\title{
Application of a Hybrid Navier-Stokes Solver with Automatic Transition Prediction
}

\author{
Andreas Krumbein* \\ Deutsches Zentrum für Luft- und Raumfahrt - DLR (German Aerospace Center), Göttingen, Germany \\ Normann Krimmelbein ${ }^{\dagger}$ \\ Technical University of Braunschweig, Braunschweig, Germany \\ Géza Schrauf* \\ Airbus, Bremen, Germany
}

\begin{abstract}
A hybrid Reynolds-averaged Navier-Stokes solver, a laminar boundary layer code and a fully automated local, linear stability code were coupled in order to predict the laminar-turbulent transition due to TollmienSchlichting and cross flow instabilities using the $\mathrm{e}^{N}$-method based on the two $N$ factor approach. The coupled system was designed to be applied to three-dimensional aircraft configurations which are of industrial relevance. The application of the system to a two-dimensional two-element airfoil configuration and a threedimensional generic full aircraft configuration is described and documented in this paper. The prediction of the laminar-turbulent transition lines was done in a fully automatic manner. It will be shown that complex aircraft configurations can be handled without a priori knowledge of the transition characteristics of the specific flow problem. The computational results are partially compared to experimental data.
\end{abstract}

\section{Nomenclature}

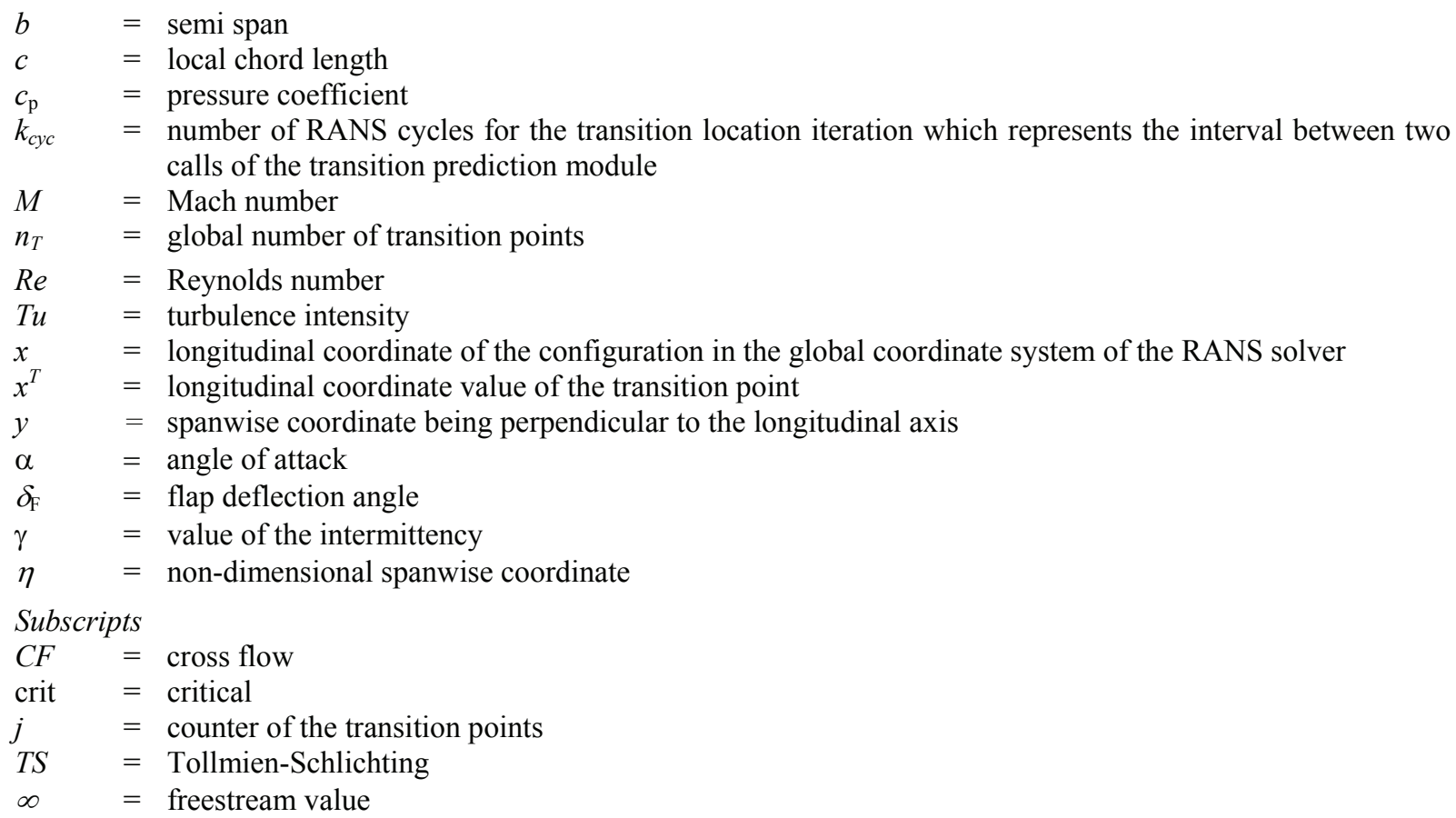

\footnotetext{
*Research Engineer, Institute of Aerodynamics and Flow Technology, Numerical Methods, Bunsenstraße 10, D-37073 Göttingen, andreas.krumbein@dlr.de, AIAA Member.

${ }^{\dagger}$ Research Engineer, Institute of Fluid Mechanics, Aerodynamics of Aircraft, Bienroder Weg 3, D-38106 Braunschweig, Germany, n.krimmelbein@tu-braunschweig.de.

‡esearch Scientist, Laminar Flow Expert, Hünefeldstraße 1-5, D-28199 Bremen, Germany, geza.schrauf@airbus.de, AIAA Senior Member.
} 


\section{Introduction}

$\mathrm{B}^{\mathrm{e}}$ esides wind tunnel testing and flight tests, computational fluid dynamics (CFD) based on Reynolds-averaged Navier-Stokes (RANS) solvers has become a standard design approach in industry, for example, for the design of transport and fighter aircraft or for helicopters. For the design point of aircraft a positive assessment of the numerical results could be achieved for many validation and application tests and the prediction capabilities could be positively evaluated. As a consequence, high confidence in numerical simulations could be achieved in industry and will eventually allow more simulation and less physical testing.

However, and despite of the progress that has been made in the development and application of RANS-based CFD tools, there is still the need for improvement, for example, with regard to the reduction of the computational costs, on the one hand, or concerning the capability of a proper capturing of all physical phenomena which can be present, on the other hand. The latter can only be tackled if capable and accurate physical models are available in the codes.

In aircraft industry and in research institutions, there exist increasing demands on CFD tools and the code users wish to have powerful and reliable turbulence and transition models at hand. On the one hand, highly accurate numerical solutions for configurations of ever increasing geometrical complexity are wanted, on the other hand, the designer's attention is turning to problems at the borders of the flight envelope or to configurations which are characterized by strongly deflected or moving control surfaces or by flow control mechanisms. For all these application areas, the combined use of turbulence and transition models is indispensable, because otherwise it is not possible to fully exploit the high potential of advanced turbulence models or to reproduce the close interaction between the laminar-turbulent transition and its interaction with flow separation.

Especially the simulation of flows around high-lift systems of aircraft may result in significant errors when the transition points are simply estimated or are not taken into account at all. High-lift systems very often involve multicomponent wings (e.g. slat, main wing, and flaps) and usually exhibit very high levels of total circulation. Because all components of the high-lift system are in close interaction with one another the total circulation and the complete flow field is affected by one transition line on one of the components.

Although the overall lift value may be predicted with satisfactory accuracy slight deviations between the real and the computed pressures can lead to large errors in the computed overall drag value. This issue was investigated in detail in [1] and it was shown that the overall pressure drag of a high-lift configuration, which dominates the drag value of the configuration as a whole as well as the drag of every single element, is composed of a balance of very large positive and negative contributions. The contribution of one single element may be one order of magnitude larger than the resulting overall drag of the complete configuration. Thus, a relative error of $5 \%$ of the computed drag on the slat upper side may result in a change of $50 \%$ for the overall drag value. This effect occurs, for example, due to suction peaks at the noses of the wing elements which do not reach the desired high pressure levels at large angles of attack, so that the suction force is too low and leads to a change of the overall drag on the one hand. On the other hand, the location of separation and the extent of the separation region are sensitive to the location of the laminar-turbulent transition lines, because separation depends on the upstream history of the boundary layer.

Another aspect of taking into account transition is, that in many cases the high potential of higher order turbulence models can be made use of only when the areas of laminar-turbulent transition are known and deployed in the computational procedures with sufficiently high accuracy. Thus, in modern CFD tools a robust transition modeling must be established together with reliable and effective turbulence models. Only if the transition locations are taken into account with sufficient accuracy all physical characteristics of the flow field can be reproduced in such a way that the demanding quality requirements of the industry are satisfied.

For the design process of wings in industry, there exists the demand for a RANS-based CFD tool that is able to handle laminar-turbulent transition automatically and autonomously during the ongoing computation. Existing transition prediction methods vary from empirical transition criteria via the local, linear stability equations based on small disturbance theory or non-local, linear and non-local, non-linear stability methods using the parabolized stability equations over large eddy simulations to direct numerical simulations of the Navier-Stokes equations. Empirical transition criteria and the $\mathrm{e}^{N}$-method [2],[3] based on local, linear stability theory and the parallel flow assumption, represent state-of-the-art methods for the prediction of transition onset in many industrial applications. Although they do not account for a number of fundamental aspects in the transition process $\mathrm{e}^{N}$-methods are used in aircraft industry most frequently for design purposes covering transition due to Tollmien-Schlichting (TS) and cross flow (CF) instabilities. Because there are no other practical methods presently available for industrial applications [4] $\mathrm{e}^{N}$-methods together with the two- $N$-factor method and empirical criteria for transition mechanisms which are not covered by the $\mathrm{e}^{N}$ approach (e.g. bypass and attachment line transition) will continue to be used for the design of 
aircraft wings and wing systems. These methods are also foreseen for future laminar wing design of transport type aircraft.

The first steps towards the setup of a RANS-based CFD tool with automatic transition prediction were made, for example, in [5], where a RANS solver and an $\mathrm{e}^{N}$-method were applied and in [6], where a RANS solver, a laminar boundary-layer method [7], and an $\mathrm{e}^{N}$-method were coupled. There, the boundary-layer method was used to produce highly accurate laminar, viscous layer data to be analyzed by a linear stability code. Hence, the very expensive grid adaptation necessary to produce accurate viscous layer data directly from a structured Navier-Stokes grid was avoided. The use of $\mathrm{e}^{N}$-database methods [8],[9] results in a coupled program system that is able to handle transition prediction automatically. In [10] a database for the instability growth rates is used which are represented by a trained neural network based on Falkner-Skan-Cooke profiles. Alternative approaches using a transition closure model or a transition/turbulence model directly incorporated into the RANS solver are documented in [11]-[13]. A correlationbased transition modelling approach built on local variables using transport equations for the intermittency and for a transition onset criterion in terms of the momentum thickness Reynolds number is documented in [14]. Recent advances in predicting transition onset in complex flows address the prediction of unsteady transition on moving airfoils [15] and the application to two-dimensional laminar separation bubbles [16]-[18]. Increasing focus is placed on the prediction of transition in three-dimensional boundary-layers on high-aspect ratio wings and high-lift systems [18]-[21], flows around bodies of revolution [22], and general three-dimensional aircraft configurations [23]-[24].

Recently the unstructured/hybrid RANS solver TAU [25]-[28] of the Deutsches Zentrum für Luft- und Raumfahrt, German Aerospace Center (DLR) has been provided with a general transition prediction functionality which can be applied to general three-dimensional aircraft configurations. The developments and first technical validation steps were carried out at the Institute of Fluid Mechanics of the Technical University of Braunschweig, [24],[29]-[30]. The TAU code is used together with the laminar boundary-layer method in [31] and the local linear stability code in [32]. These two codes and an infrastructure part of the TAU code form a so called 'transition prediction module' that is coupled to the RANS solver and that interacts with the RANS solver during the computation in a very similar way as it is documented in [19]-[21].

For a long time it was necessary to use transition database methods in order to apply the $\mathrm{e}^{N}$-method for transition prediction in a fully automatic way, so that the transition location iteration could be executed without intervention (automatic) by the user of the RANS code and without a priori knowledge of the transition characteristics of the specific flow problem (autonomous). Now the fully automated local, linear stability solver in [32] is available using a frequency estimator for the detection of the relevant regions of amplified disturbances for TS instabilities and a wave length estimator for $\mathrm{CF}$ instabilities and can now be used instead of the $\mathrm{e}^{N}$-database methods which have a more limited application range.

The objective of this paper is the demonstration of the transition prediction functionalities of the TAU code when it is applied to aerodynamic configurations of industrial relevance and the documentation of the different application modes in order to show that the coupled system can be used by the design engineer for flow problems in which laminar-turbulent transition plays a role. The TAU code is applied to a two-dimensional two-element airfoil configuration and a three-dimensional generic full aircraft configuration using the integrated automatic transition prediction procedure. The coupling structure between the TAU solver and the transition prediction module is outlined, the functionalities of the transition prediction module are described, the test cases and the computational results are presented and partially compared to experimental findings.

\section{Transition Prediction Coupling Structure}

The DLR TAU code is a three-dimensional, compressible RANS code for steady or unsteady flow problems and uses unstructured or hybrid grids, which may consist of hexahedral, tetrahedral, pyramidal, and prismatic cells and, therefore, combine the advantages of regular grids for the accurate resolution of the viscous shear layers in the vicinity of walls with the flexibility of grid generation techniques for unstructured grids. The use of a dual grid makes the solver independent of the type of cells that compose the primary grid. The primary grid describes the geometry and the discretized space around the geometry based on the outcome of a grid generation tool. The dual grid is derived from the primary grid and forms the control volumes through which the fluxes are computed. The code is based on a finite volume formulation and uses an edge-based dual-cell approach, i.e. a vertex-centered scheme, where the inviscid terms are computed employing either a second-order central scheme or a variety of upwind schemes using linear reconstruction (of the left and right states) for second-order accuracy. The viscous terms are computed with a second-order central scheme. Scalar or matrix artificial dissipation may be chosen by the user and a low Mach number preconditioning is available enabling the solver to be applied to incompressible flow 
problems. The time integration can be carried out using either an explicit hybrid multistage Runge-Kutta scheme or an implicit approximate factorization scheme (LU-SGS: Lower-Upper Symmetric Gauss-Seidel). For steady-state calculations, the integration is accelerated by local time stepping and explicit residual smoothing. These techniques are embedded in a multigrid algorithm based on agglomerated coarse grids. For time accurate computations the dual time stepping approach of Jameson is employed. The influence of turbulence is taken into account either by oneequation or two equation eddy viscosity turbulence models according to the Boussinesq approximation or by algebraic or differential Reynolds stress models. The fixing of prescribed transition lines on the surfaces of the geometry can performed for general three-dimensional configurations.

The transition prediction module coupled to the TAU code consists of an infrastructure part inside the RANS solver and a number of external codes which are used for transition prediction purposes. The infrastructure part processes geometrical data on the surfaces of the configuration, for example, the extraction of the surface $c_{p^{-}}$ distribution or the computation of inviscid streamlines and the corresponding laminar boundary-layer parameters. In the latter case, the laminar boundary-layer parameters are directly computed inside the RANS computational grid and, thus, their accuracy depends on the grid and its quality. This dependency does not appear if the laminar boundary-layer parameters are provided by the boundary-layer code using the surface $\mathrm{c}_{p}$-distribution as input. The external tools are a streamline-oriented, laminar boundary-layer method for swept, tapered wings [31] and different transition prediction methods, which are provided with all necessary boundary-layer data either by the laminar boundary-layer method (BL mode 1) or by the infrastructure part of the transition prediction module that determines the boundary-layer data from the current flow solution in the RANS grid (BL mode 2). The laminar boundary-layer method solves the compressible, laminar boundary-layer equations for conical, external flow. Besides a number of empirical transition criteria and two $\mathrm{e}^{N}$-database methods for Tollmien-Schlichting (TS) instabilities [8] and for cross flow (CF) instabilities [9], the most general transition prediction method that is currently available is a fully automated linear, local stability code named LILO [32]. The LILO code uses a frequency estimator for the detection of the relevant regions of amplified disturbances for TS instabilities and a wave length estimator for CF instabilities. The code applies $\mathrm{e}^{N}$-methods for TS and CF waves according to the two $N$ factor approach [33]-[37].

For BL mode 1, the RANS solver communicates the surface $\mathrm{c}_{p}$-distribution of the configuration as input data to the laminar boundary-layer method, the laminar boundary-layer method computes all of the boundary-layer parameters that are needed for the transition prediction methods. For BL mode 2, the RANS solver communicates the internally computed laminar boundary-layer parameters directly to the transition prediction methods. Then, the transition prediction methods determine new transition locations that are given back to the RANS solver.

This coupled structure results in an iteration procedure for the transition locations within the iterations of the RANS equations. The structure of the approaches using the two different BL modes is outlined graphically in Fig. 1.

During the computation, the RANS solver is stopped after a certain number of iteration cycles, $k_{c y c}$, usually when the lift has sufficiently converged, that is when pressure oscillations have been damped to

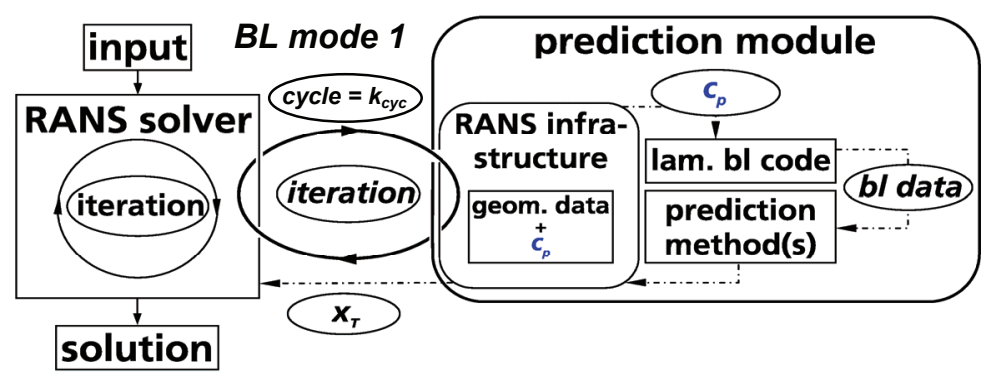
a sufficiently low degree. Then the

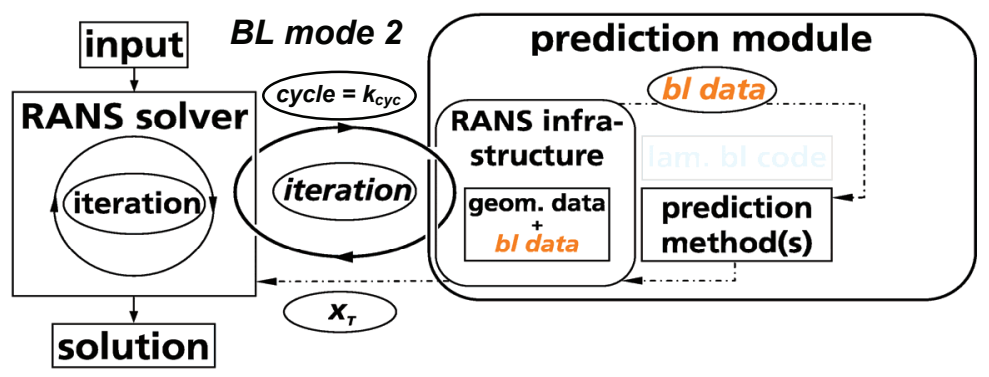

Fig. 1 Coupling structure of the RANS solver and the transition prediction module.

transition module is called, the geometrical data are processed and all laminar viscous data - basically the velocity profiles in streamwise and crossflow direction and their $1^{\text {st }}$ and $2^{\text {nd }}$ derivatives - are calculated either by the BL code or by the TAU code itself. Then, either the two $\mathrm{e}^{N}$-database methods or the stability code analyze the laminar boundary layer and try to determine a transition point. For BL mode 1 this is possible only when the transition point is located upstream of the separation point predicted by the boundary-layer code, because the boundary-layer code terminates when a separation is detected. If a transition point due to TS or CF instabilities was found it is 
communicated back to the RANS solver. If no transition point due to TS or CF instabilities upstream of the laminar separation point could be found the laminar separation point is used as approximation of the real transition point. This is an attempt to predict transition due strictly to the presence of separation bubbles. This approach often yields a good approximation of the real transition point when transition does not occur before the laminar boundary layer separates, particularly for low Reynolds number flows. For BL mode 2 the laminar BL data are calculated beyond the point of laminar separation which is detected by the TAU code inside the RANS computational grid. Thus, transition inside laminar separation bubbles can be detected without relying on an approximation.

These steps are done for the upper and lower sides of all specified wing sections. When all new transition locations, $x_{j}^{T}$ (cycle $=k_{c y c}$ ) with $j=1, \ldots, n_{T}$, where $n_{T}$ is the number of transition points, have been communicated back to the RANS solver, each transition location is slightly underrelaxed to damp oscillations in the convergence history of the transition locations. Then, all underrelaxed transition points - they represent a transition line on the upper or lower surface of a wing element in form of a polygonial line - are mapped onto the surface grid of the configuration applying a transition setting algorithm subdividing the surface of the geometry into laminar and turbulent regions, and the computation is continued. In so doing, the determination of the transition locations becomes an iteration process itself. With each transition location iteration step the underrelaxation factor is reduced until a converged state of all transition points has been obtained. In the last prediction step no underrelaxation is applied, so that the transition point value from the prediction method is considered directly and without manipulation in the following phase of the RANS computation.

Using BL mode 1, the application of a laminar boundary-layer method for the computation of all viscous data necessary for the transition prediction methods ensures the high accuracy of the viscous data required by the $\mathrm{e}^{N}$ methods for the analysis of laminar boundary layers. Thus, as shown in [6], the large number of grid points near the wall for a high resolution of the boundary layers, the adaptation of the Navier-Stokes grid in the laminar, turbulent and transitional boundary-layer regions and the generation of new adapted grids for the RANS solver after every step of the transition location iteration are avoided and the computational time can be massively reduced. In addition, the number of RANS iteration cycles between two steps of the transition location iteration can be highly reduced compared to an approach where the boundary-layer parameters are computed directly from the RANS grid [38]-[39], because the surface pressure converges significantly faster in the RANS computation than the boundarylayer velocity profiles [24],[29]-[30] which are the basis for the computation of the boundary-layer parameters. Because the laminar separation point is used as an approximation of the real transition point in the case that the $\mathrm{e}^{N}$ methods do not detect transition upstream of the separation, this approach may fail when transition occurs inside a laminar separation bubble. In such a situation, the application of empirical or semiempirical criteria for a better approximation of the transition point is possible. These criteria which are based on information at the laminar separation point itself add an additional distance to the location of laminar separation and, thus, shift the transition point beyond the separation point [40]-[41].

For BL mode 2, the laminar boundary-layer data are calculated downstream of the point of laminar separation which is detected by the TAU code inside the RANS computational grid. Thus, transition inside laminar separation bubbles can be detected without relying on an approximation. Practically, the determination of transition inside laminar separation bubbles is only realizable using the linear, local stability solver because the $\mathrm{e}^{N}$-database methods lack parameters for the base flow profiles in laminar separation bubbles. The use of this mode implies that the laminar separation bubble is well resolved by enough grid points in wall normal direction, because otherwise the bubble characteristics are not reproduced well enough in the boundary-layer profiles on the one hand. In contrast to a purely structured computational grid, however, the number of grid points, which are additionally necessary compared to BL mode 1, is limited to the prismatic cell layer which appears structured in the hybrid grid. Thus, the additional computational effort coming from the handling of more unknowns in the linear equation system of the RANS solver is tolerable. On the other hand, the calculation of the laminar boundary-layer data from the RANS grid leads to low quality boundary-layer profiles for the cross flow direction. Whereas for an accurate representation of the streamwise velocity profile including its $1^{\text {st }}$ and $2^{\text {nd }}$ derivatives 48 cells in the prismatic grid layer were sufficient for a proper stability analysis with respect to TS waves, 128 cells were needed for the same accuracy of the cross flow velocity profile and its corresponding derivatives in a test case problem in [24],[29]-[30]. For threedimensional configurations of some complexity or for really complex configurations as they are usual today in industry, this requirement which is based on the approach that the prismatic layer contains the complete laminar as well as the complete turbulent boundary layers in an area with constant wall normal extent, can not be satisfied. To overcome this problem, the application of the adaptation functionality of the TAU code [28] is planned. Starting from a normal prismatic layer with constant wall normal extent an adaptation procedure would adapt the outer edge of the prismatic layer in the region of laminar flow according to the laminar boundary-layer thickness which is available anyway for each surface grid point. The initial wall-normal point distribution inside the prismatic layer 
which is made by default for a turbulent boundary layer and, thus, exhibits a near wall clustering of the cells, would be changed in such a way that the gradients of a laminar boundary-layer profile, which are located some distance apart from the wall, are accurately resolved. This can be achieved by a more or less equidistant wall-normal point distribution [42]. An accurate reproduction of all cross flow profiles can be achieved distributing about 70 grid points between the surface and the laminar boundary-layer edge. In so doing, about $45 \%$ of the additional points necessary for the high quality resolution of the cross flow profiles can be saved.

For a proper resolution of a laminar separation bubble, regardless of which BL mode is applied, sufficiently many points around the contour of the wing section are to be distributed in order reproduce the location of the laminar separation and the extent of the separation bubble as good as possible.

The definition of a wing section is done using two different approaches which can be selected by the user of the code. The first approach (WS mode 1) applies 'line-in-flight' cuts, that is, the wing is cut through parallel to the longitudinal direction of the configuration in the global coordinate system of the RANS solver, according to strip theory. The second approach (WS mode 2) uses the boundary-layer edge streamlines running over the upper and lower sides of the wing originating in the boundary-layer edge point associated with the stagnation point (in twodimensional problems) or with the attachment line point (in three-dimensional problems) on the wing surface in the area of the oncoming flow [30]. Therefore, the attachment line is integrated using the skin friction distribution in the nose region of the wing. For the calculation of the inviscid streamlines the boundary-layer edge velocities are first projected onto the surface of the wing. Then, the streamlines are integrated.

\section{Computations}

\section{A. Two-dimensional two-element configuration}

In the work presented here, the TAU code with integrated automatic transition prediction was first applied to the two-dimensional two-element airfoil configuration from [43] consisting of a main airfoil with a single-slotted trailing edge flap. The main airfoil is based on the supercritical NLR 7301 airfoil and the flap whose chord length is $34 \%$ of the main airfoil's chord length, $c=0.57$ meters, has a flap deflection angle of $\delta_{\mathrm{F}}=20 \mathrm{deg}$. The gap width between the main airfoil trailing edge and the flap upper surface is $2.6 \% c$.

The configuration is depicted in Fig. 2 showing the primary hybrid grid as it comes from the grid generator. The grid consists of about 23,000 triangles and about 15,000 quadrilaterals in the prismatic layers with 250 points around the main airfoil contour and 180 point around the flap contour. The prismatic layers contain 36 cells in

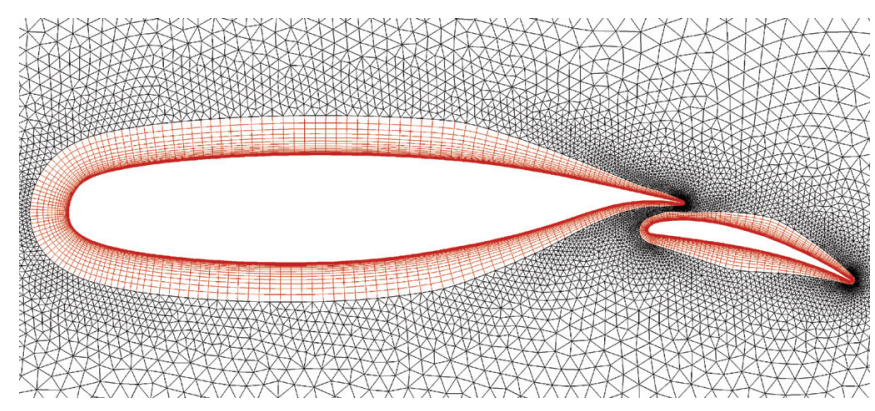

Fig. 2 Computational grid of the two-dimensional twoelement test case.

wall normal direction. The computations were carried out for the Reynolds number $R e_{\infty}=1.35 \times 10^{6}$ based on the chord length of the main airfoil, the Mach number $M_{\infty}=0.185$ and the angle of attack $\alpha=6.0$ deg using the SpalartAllmaras one-equation turbulence model [44] with Edwards and Chandra modification [45] (Spalart-AllmarasEdwards $=\mathrm{SAE}$ ). Based on the information in [43], the following regions for separation or transition respectively both phenomena were detected by flow visualization based on napthalene sublimation - are given:

\begin{tabular}{lcc}
\hline \hline & upper side & lower side \\
\hline \multirow{2}{*}{ main } & separation: & transition: \\
& $\left(x^{\text {sep }} / c\right)^{\text {main }}=0.027$ & $\left(x^{\text {Tstart }} / c\right)^{\text {main }}=0.65$ \\
& $\left(x^{\text {attach }} / c\right)^{\text {main }}=0.04$ & $\left(x^{\text {Tend }} / c\right)^{\text {main }}=0.68$ \\
& transition: & \\
flap & $\left(x^{\text {Tstart }} / c\right)^{\text {flap }}=0.606$ & - \\
& $\left(x^{\text {Tend }} / c\right)^{\text {flap }}=0.644$ & \\
\hline \hline
\end{tabular}

Table 1: Separation and transition regions of the two-dimensional two-element test case. 
The critical $N$ factor for TS instabilities which was applied together with the linear, local stability code LILO was set to $N^{T S}$ crit $=9.0$, which corresponds to a free-stream turbulence intensity of $T u_{\infty} \approx 0.07 \%$ according to Mack's relationship, $N_{\text {crit }}^{T S}=-8.43-2.4 \ln \left(T u_{\infty}\right)$.

Fig. 3 shows the convergence history of the transition location iteration for the upper and lower sides of the two elements of the configuration when BL mode 1 is applied, that is, the laminar-boundary layer parameters are calcu-

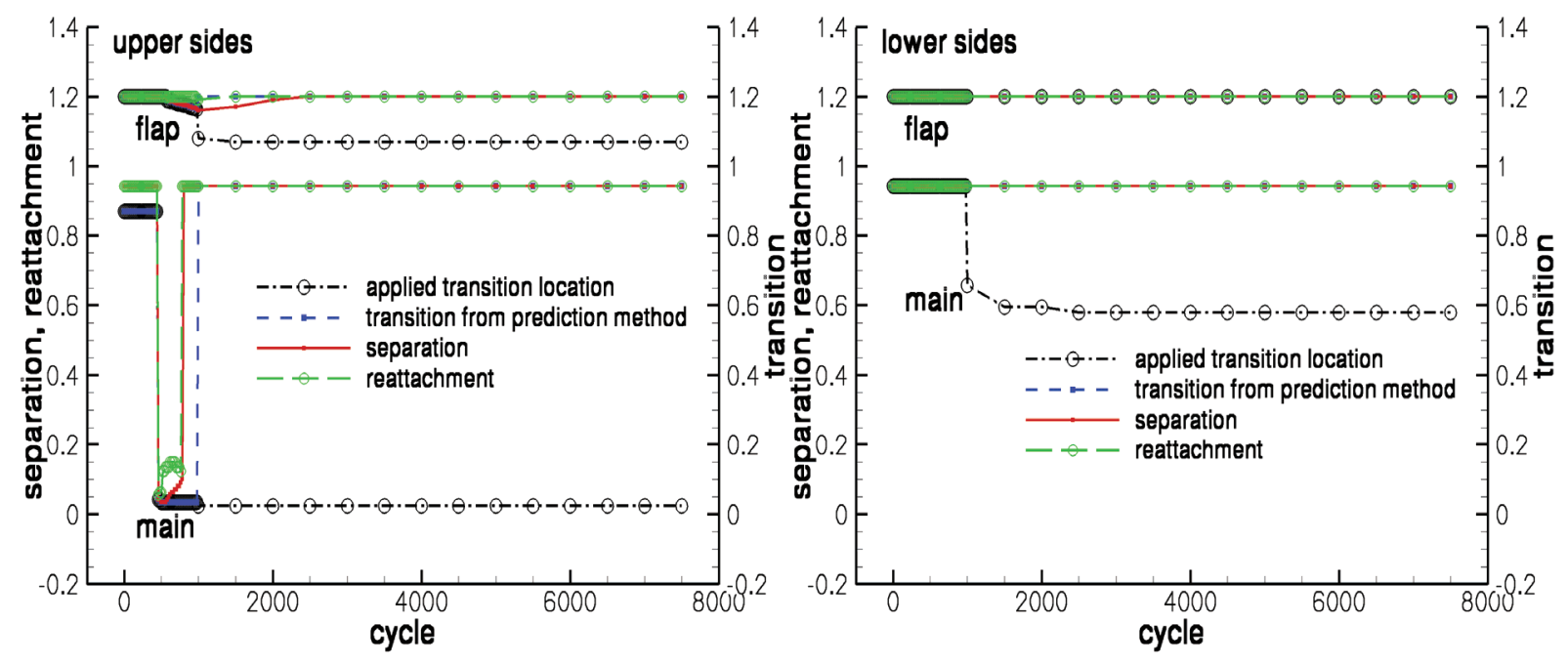

Fig. 3 Convergence history of the transition locations for the two-element airfoil, BL mode 1.

lated by the laminar boundary-layer code. During the first 1000 iteration cycles of the RANS computation every 20 cycles the RANS solution is checked for laminar separation points which are immediately used as transition points. The calculation was started with initial transition locations set at the trailing edges of the two elements, so that a fully laminar flow is computed in the beginning. After about 500 RANS cycles, laminar separation points occur on the upper sides of the two elements for the first time. A laminar separation bubble then evolves on the main airfoil upper side. This bubble degenerates to a turbulent bubble and finally vanishes. On the flap upper side, the laminar separation is moving continuously upstream while exhibiting a separated region up to the flap trailing edge. In the figures, the location of a separation point is denoted by a solid line with squared symbols, the location of a reattachment point by a long-dashed line with circles. A transition location is denoted either by a dashed-dotted line with circles or with a dashed line with squares depending on which component of the transition prediction module has detected the current transition point. The explanation of these two different depictions of the convergence of the transition locations follows in the next section. In this phase of the transition prediction iteration - the pre-prediction phase, which ends when the transition prediction module is called for the first time - there exists no distinction between these two different depictions.

At RANS cycle $k_{c y c}=1000$, the transition prediction module was called for the first time and from this point on it was called again every 500 RANS cycles. The laminar boundary-layer code detects laminar separation points on the upper sides of the main airfoil and the flap and on the main lower side. All laminar separation points are used as transition points, because the stability code does not find amplified TS waves which could lead to transition upstream of the laminar separation points. After four calls of the transition prediction module, the laminar separation points have settled in all cases and do not change anymore. Meanwhile, the separation that existed on the flap upper side has disappeared and the flow over the flap is fully attached on both sides. The flow on the flap lower side stays fully laminar. After the fourth prediction step the iteration of the transition points has converged and the transition location iteration could have been stopped. The dashed line with squares depicts the transition point values which are determined by the linear stability code. Because the stability code does not detect transition in this simulation the dashed line with squares jumps back to the trailing edge point on the two upper sides of the two airfoil elements and thus indicates that no transition points due to TS instabilities were found. The dashed-dotted line with circles depicts either the underrelaxed transition point value if the transition point were due to a TS instability and detected by the stability code or - and that is the situation here for all cases - laminar separation points from the boundary-layer code which approximate real transition points which in reality are located downstream of a laminar separation point.

In Fig. 4, all predicted transition locations (hollow circles), the laminar regions for both airfoil elements (dashdotted lines) and the computed $c_{\mathrm{p}}$-field are shown. The experimentally observed transition regions are reduced to 
single transition points by taking the coordinate value in the middle of the particular transition region and depicted by filled circles.

When BL mode 2 is applied, it is not as easy to reach a well converged state of the transition locations as for BL mode 1. Fig. 5 shows the convergence histories for the case that a transition prediction step is carried out every 1000 cycles. Again, the transition prediction module was called at RANS cycle $k_{c y c}=1000$ for the first time. Now, in the RANS grid laminar separations evolve on the main upper side, on the flap upper side, and on the main lower side. Whereas the laminar separation points have settled on the main upper side after the second call of

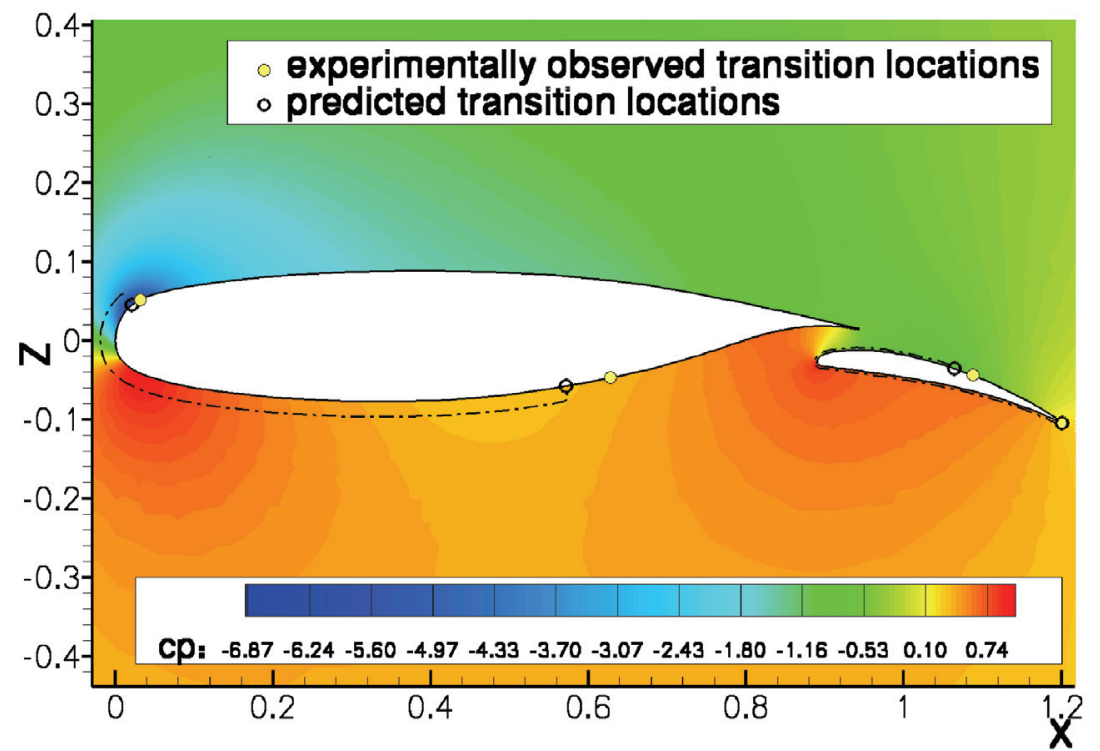

Fig. 4 Transition locations, laminar regions, and $c_{\mathrm{p}}$-field for the twoelement airfoil, BL mode 1.

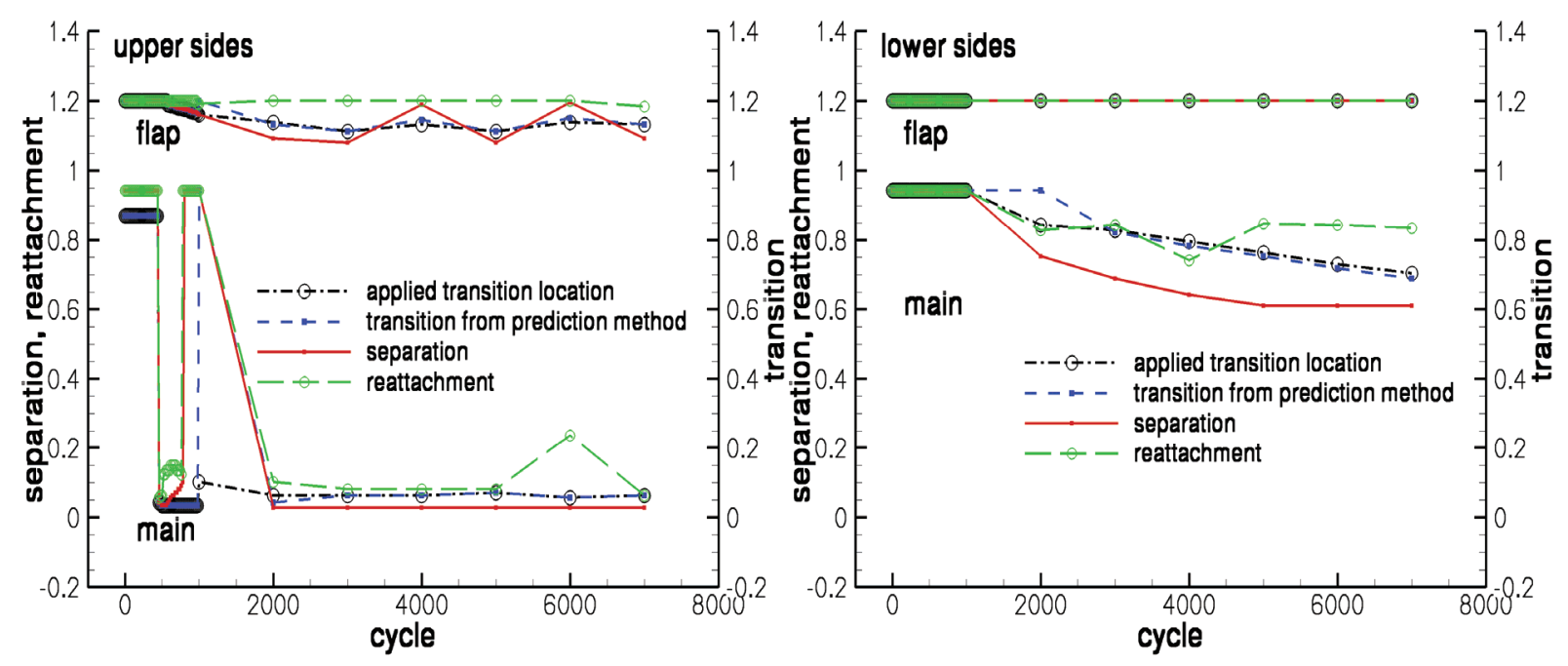

Fig. 5 Convergence history of the transition locations for the two-element airfoil, BL mode 2, transition module called every 1000 cycles.

the transition prediction module and on the main lower side after the fifth call, the laminar separation point on the flap upper side exhibits oscillations. Downstream of all laminar separation points bubbles have evolved and the stability code has determined transition points which are in well converged state on the upper side of the main airfoil and the flap after the third call of the transition module. The transition point on the flap upper side is affected slightly by the oscillation of the laminar separation point. The transition point on the main lower side has not yet converged due to a limiter that acts on the maximum difference between two consecutive underrelaxed transition points which arise in the iteration procedure. Here, the setting for this limiter was $10 \%$ of the corresponding airfoil's chord length. Keeping this value of the limiter and simply increasing the maximum number of module calls through a later shutdown of the transition module - shutdown at $k_{c y c}=10000$, instead at $k_{c y c}=7000$ - leads to the situation in Fig. 6. After the ninth call of the transition module the transition point on the main lower side has converged and the laminar separation point has settled. On the main upper side, however, the laminar separation point has vanished, the stability code does not find amplified disturbances which exceed the critical $N$ factor and the transition point is moving downstream controlled by the limiter setting. If one increases the number of module calls further by reducing the interval length between two calls, so that the transition module is called every 500 cycles, the situation 

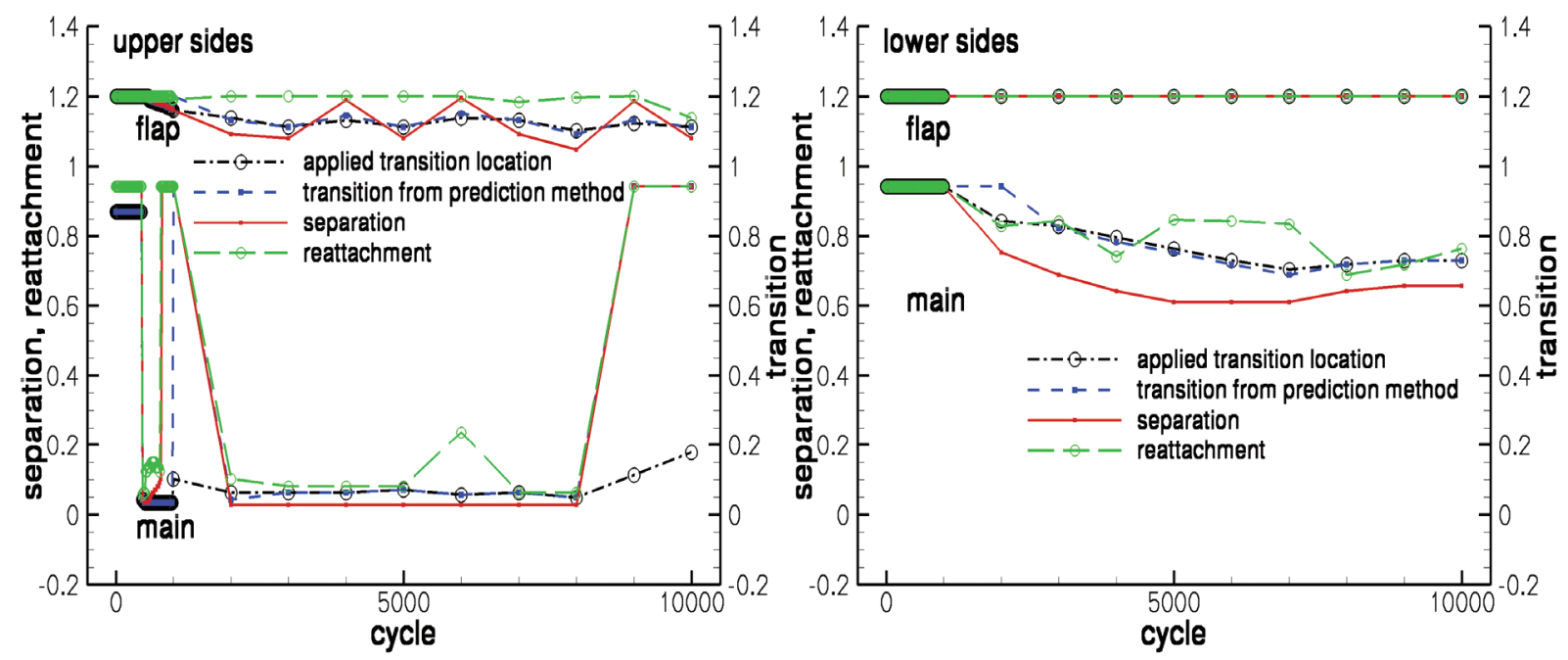

Fig. 6 Convergence history of the transition locations for the two-element airfoil, BL mode 2, transition module called every 1000 cycles, longer computation time.

depicted in Fig. 7 occurs. On the flap upper side and the main airfoil lower side the convergence behaviour is very similar as in the two cases before. On the main airfoil upper side, one finds an intermediate oscillation of the laminar separation point and the corresponding transition point. After the sixteenth call of the transition module this oscillation has disappeared. The oscillation on the flap upper side, however, remains. It must be pointed out, that the separation points which are located furthest upstream have always the same coordinate value throughout the oscillations and that, in principle, the separation points on the upper sides of both elements have settled and the corresponding transition points have converged after the fifth call of the transition module. At this point, the transition location iteration for the upper sides should have been stopped. The functionality of an individual, automatic shutdown of the transition location iteration for a single airfoil side or wing section side must and will be
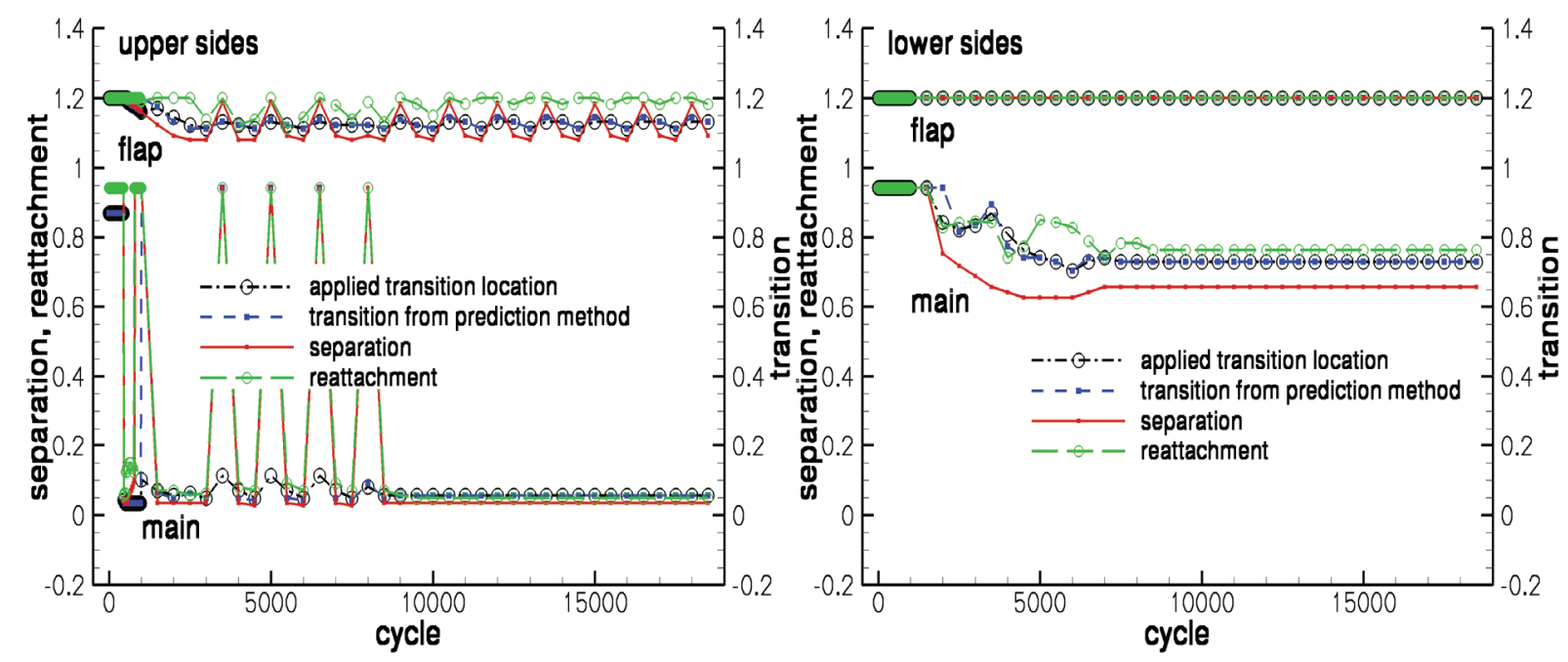

Fig. 7 Convergence history of the transition locations for the two-element airfoil, BL mode 2, transition module called every 500 cycles, longer computation time.

incorporated into the transition prediction module in the nearest future.

Based on the information from [43], where it is reported that on the flap upper side no laminar separation bubble was observed in the experiment, and taking into account the convergence histories of the transition locations as well as the RANS convergence history of the computation which belongs to the results in Fig. 5 and which is depicted here in Fig. 8, the oscillations can be explained as follows: At first, in the transient phase of the computation, laminar separations evolve on the upper sides of the main airfoil and on the flap due to the fact that the initial transition points were fixed at the trailing edges, which does not reflect the real physical situation. The RANS solver 
reacts to this defect by producing laminar separations. Because the separation points are immediately used as transition points, the computation remains stable and the disadvantageous influence of the wrong initial transition locations diminishes. Whereas on the main upper side the laminar separation point has reached its final position in the very beginning of the pre-prediction phase and then disappears, the upstream movement of the flap upper side laminar separation point proceeds very slowly. When the first transition prediction step is carried out, no separation exists on the main upper side, the stability code can not find amplified disturbances, and the current transition location is shifted a bit downstream according to the limiter setting. On the flap upper side, the upstream movement of the laminar separation point continues. In the second prediction step, a new laminar separation is found on the main upper side and the stability code detects transition slightly downstream of the laminar separation point. On the flap upper side, the upstream movement of the laminar separation point continues and the stability code detects transition near the trailing edge. After some calls of the transition module the laminar separation points have settled on the upper sides of both elements and the transition points seem to have converged. This situation is labeled with ' 1 ' in Fig. 8. In the next prediction step, the laminar separation on the flap upper side has vanished, because the current transition location is a good approximation of the real transition point and separation does not occur in the real flow problem. This is reproduced here by the simulation. Now, without separation, the stability code finds a new transition point which is located two surface grid points downstream of the last one and the new transition location is fixed there. The position of this new transition point seems to be very slightly too far downstream, so that a new laminar

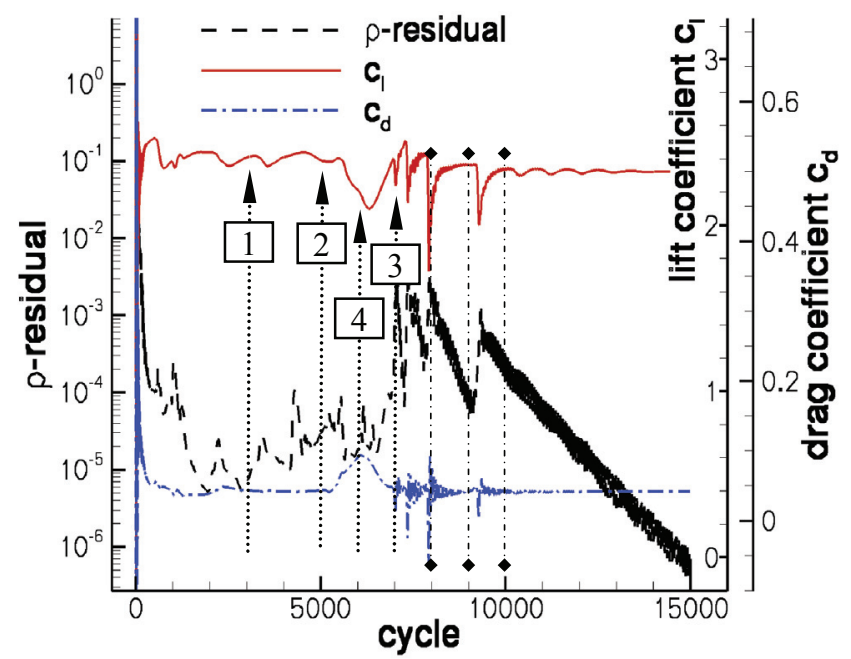

Fig. 8 RANS convergence history for the two-element airfoil, BL mode 2, transition module called every 1000 cycles.

separation can develop on the flap upper side. In Fig. 8, at the position labeled with '2', that RANS cycle is pointed at, where laminar separation at the flap is detected anew by the transition module. The position labeled with ' 3 ' marks the situation at the end of the transition prediction phase shown in Fig. 5. From this point on, the transition locations are fixed. In between, the separation bubble on the main upper side grew in size and shrank to its old size again. The position labeled with ' 4 ' marks the situation where the separation bubble on the main upper side has grown to a size of about $20 \%$ of the main airfoil chord length as shown in Fig. 5. Now, the computation continues with fixed transition locations and, at first, the lift climbs. At cycle $k_{c y c} \approx 7300$, the lift breaks down, see Fig. 8. This occurrence is not reflected in the convergence history shown in Fig. 6, because the transition module was not called around this RANS cycle. Before $k_{c y c}=8000$, the lift has time to recover, but can not climb as high as it was before at $k_{c y c} \approx 7300$, see Fig. 8. Some ten cycles before $k_{c y c}=8000$, the lift breaks down again. Somewhere here, the laminar separation on the flap upper side must have moved further upstream, as it is reflected in Fig. 6 at cycle $k_{c y c}=8000$. In this situation, the bubble on the main airfoil upper side has slightly grown and the separation on the flap upper side has its largest size in this computation, as shown in Fig. 6. These effects provoke the lift breakdown which occurs before $k_{c y c}=8000$, that is, before in the elongated computation the new transition points were determined and set. From this point on, two more significant lift breakdowns occur in the phase

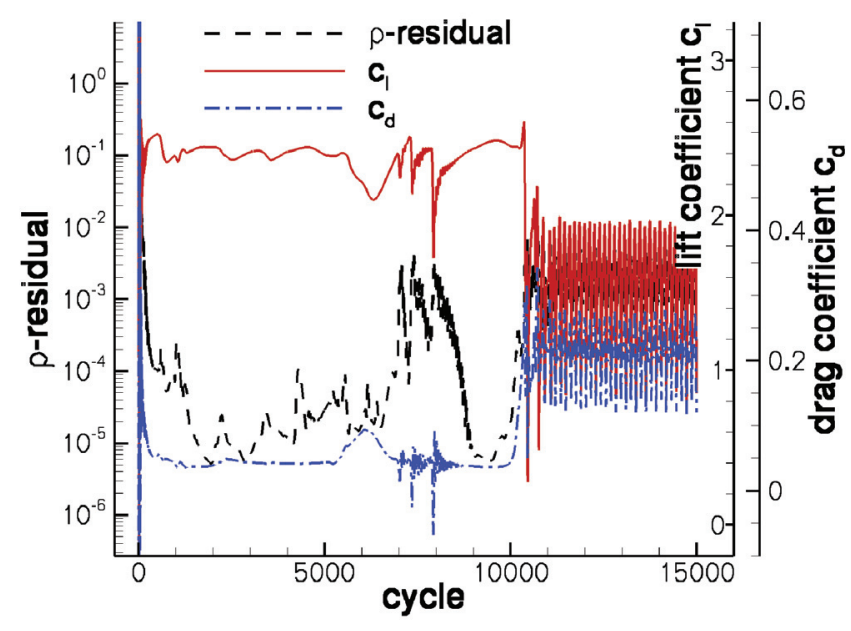

Fig. 9 RANS convergence history for the two-element airfoil, BL mode 2, transition module called every 1000 cycles, longer computation time.

10 
of the computation where the transition locations are fixed. These two more breakdowns are followed by a number of smaller breakdowns. Here, another effect comes into play: the disappearance and recurrence of the separation on the main upper side. A look at the RANS convergence history in Fig. 9, which belongs to the elongated computation whose transition prediction convergence is shown in Fig. 6, reveals the following: Because of the identical computation settings, the convergence history from Fig. 9 is the same as the one in Fig. 8 until RANS cycle $k_{c y c}=$ 8000. Between $k_{c y c}=8000$ and $k_{c y c}=9000$, the new transition locations from $k_{c y c}=8000$ in Fig. 6 lead to an increase of the lift, because they are located a bit further upstream than those from the prediction step before, the separations start to become smaller and finally vanish. At $k_{c y c}=9000$, this situation is reflected in Fig. 6 and on both upper sides the transition locations are shifted downstream, on the main upper side controled by the limiter value. Between $k_{c y c}=9000$ and $k_{c y c}=10000$, the separation on the flap upper side is moving upstream again, but on the main upper side no new separation has developed. At $k_{c y c}=10000$, the transition point on the main upper side is shifted downstream once more, again controlled by the limiter value, leading to a massive separation on the main airfoil upper side at $k_{c y c} \approx 10400$ as indicated in Fig. 9. Because the transition module was shut down at $k_{c y c}=10000$, the last transition location on the main upper side was not moved upstream again and no convergence could be obtained in this computation. The disappearance of the main upper side separation point can be explained by the fact, that in the phase, when the flap upper side separation point moves downstream and, thus, the flap separation region is shrinking, the flow on the flap upper side undergoes acceleration in streamwise direction. This acceleration increases the global circulation of the configuration and, thus, also increases the circulation of the main airfoil, which, in turn, leads to an acceleration of the flow on the main

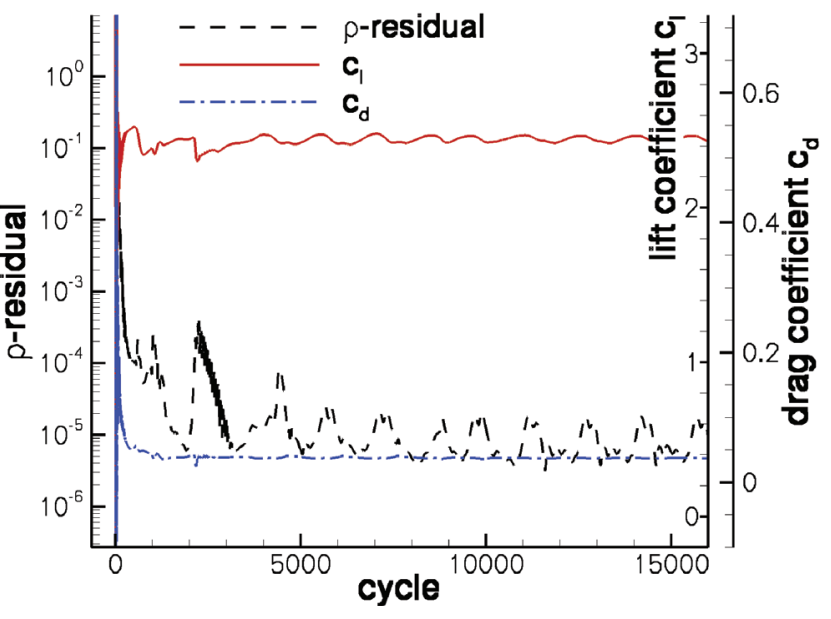

Fig. 10 RANS convergence history for the two-element airfoil, BL mode 2, transition module called every 500 cycles, longer computation time.

airfoil upper side. Due to this secondary acceleration, the disturbances which are detected by the stability code are damped there, the critical $N$ factor is not reached anymore and no transition point can be determined. An inspection of the $N$ factor curves for the main upper side for $k_{c y c}=9000$ shows, that disturbances are amplified only within the first $4 \%$ of the main airfoil chord and that the maximum value of the $N$ factor is around 1.8 . Why this does not happen before, for example, between $k_{c y c}=3000$ and $k_{c y c}=4000$ or between $k_{c y c}=5000$ and $k_{c y c}=6000$, can not be explained up to now with the current amount of information. Very probably, the occurrence of this behaviour depends strongly on the interaction between the current locations of the separation points, transition points, and reattachment points on the two elements, on the one hand, and on the global convergence level of the RANS computation, on the other hand. It has to be pointed out, that the occurrence of this behaviour is accompanied by low values of the density residual of the RANS computation and a smooth curve shape of the lift coefficient, as shown in Fig. 10, which depicts the RANS convergence history associated with Fig. 7. Here, the first occurrence of vanishing separations at the upper sides of the main airfoil and the flap, which were both monitored by the transition module during the same call of the module at $k_{c y c}=3500$, is accompanied by values of the density residual of the order of $10^{-5}$, the same order as in the computation documented in Fig. 6 and Fig. 9.

The first conclusion which can be drawn from this comparison of results is that the primary instability in this coupled computation, which is the oscillation of the flap upper side separation point, is due to the fact that in the current development state of the transition module the location of the transition point is tightly coupled to the location of the separation point and that the transition location iteration is not individually shut down for a single airfoil element or a single wing section after a certain number of iteration steps, for example, when the transition point does not change much anymore and, thus, satisfies a convergence criterion, which could be based upon the difference of two consecutive transition points. As will be shown on the next page, the transition prediction iteration is in interaction with a slight oscillation of the separation point which evolves on the flap upper side also without the application of the transition prediction procedure and which is inherently contained in the problem. However, the influence of the transition prediction procedure strongly amplifies the amplitude of this oscillation. The second conclusion which can be drawn from this comparison is that a secondary instability develops in this coupled computation, which is not 
initially induced by the transition prediction procedure, but which is inherent in this flow problem, the disappearance and recurrence of the separation on the main upper side. This second instability and the primary instability are in interaction with one another, but the second instability also appears with fixed transition locations, as shown in Fig. 8. The second instability is damped out by the coupled procedure itself.

A lot of tests were carried out using all the control parameters of the transition prediction module which are currently available in order to obtain a stable solution for this test case applying BL mode 2, but the results were not much of a difference. Finally, the results in terms of the predicted transition locations, the laminar regions and the computed $c_{\mathrm{p}}$-field based on the computations documented in Fig. 7 and Fig. 10 are shown in Fig. 11 in the same way as in Fig. 4.

In Fig. 12, the RANS convergence history from a computation with prescribed transition locations is shown. In this computation, for the main upper and lower sides the transition locations obtained from the computation documented in Fig. 7 and Fig. 10 were fixed on the surfaces using the coordinate values as they were given at the end of the transition prediction iteration shown in Fig. 7. For the flap upper side that transition point was used that has the most upstream location in the oscillation. In so doing, the strength of the laminar separation on the flap upper side turned out to be small enough, so that the occurrence of the secondary instability could be prevented. The computation was started with free stream values as initialization, as all the other computations before. As one can see, the computation converges without problems apart from the fact that after a while a long wave oscillation of the lift coefficient appears accompanied by an oscillation of the density residual. These oscillations are due to the slight oscillation of the separation point which evolves on the flap upper side and which was already mentioned before. An inspection of the flow field solutions at two different intermediate states associated with the maximum and minimum amplitude of the oscillation reveals that again a laminar separation point appears and disappears. This oscillation can not be damped by the steady solution procedure of the RANS solver together with the current parameter settings of the numerical scheme. In Fig. 12, until RANS cycle $k_{c y c}=9500$ the steady computation was carried out. Then, the computation was continued applying the dual time stepping approach for time-accurate computations using the same parameter settings of the numerical scheme as before. Now, the oscillation is damped and a steady solution is obtained. The blow-up of the curve of the lift coefficient in the upper part of Fig. 12 shows the evolution of the lift coefficient including all intermediate values of the inner iterations of the dual time stepping procedure. A blow-up of the curve of the density residual shows that 
between two physical time steps of the time-accurate computation the residual has decreased by two to three orders of magnitude.

With respect to the accuracy of the predicted transition locations using BL mode 2 it must be pointed out, that the results presented are of preliminary character, because the setting of the critical $N$ factor $N_{\text {crit }}^{T S}=9.0$ was an arbitrary choice. Until now, no information about the critical $N$ factor or the free stream turbulence intensity $T u_{\infty}$ of the wind tunnel used for the experiment [43] (NLR $3 \times 2 \mathrm{~m}$ low-speed wind-tunnel) are available to the authors. The next computations which will be carried out for this two-dimensional two-element configuration will be based on $N$ factor settings which are either correlated with the value of the free stream turbulence intensity $T u_{\infty}$ of the wind tunnel, or, at least, on a calibration of the critical $N$ factor based on one of the experimentally determined transition locations as discussed in [46]. Apart from the automatic, individual shut-down of the transition prediction procedure for a single airfoil element or a single wing section, further computations using a finer computational grid are planned. It is expected, that the observed oscillation of the flap upper side separation point does not occur at all, or that, at least, the oscillation is damped.

\section{B. Three-dimensional generic full aircraft configuration}

The next test case presented here is a three-dimensional generic full aircraft configuration represented as halfconfiguration in a hybrid grid exhibiting an overall number of grid points of about 12 million, Fig. 13. The configuration consists of the fuselage of a transport type aircraft with a high-aspect ratio wing, a vertical tail plane, and a horizontal tail plane. The wing is equipped with a deflected full span trailing edge flap. The flap deflection was realized through a rotation of the wing trailing edge region inside the computational grid, so that no gap exists between the flap and the trailing edge of the main wing.

In the prismatic layers of the hybrid grid 32 grid points were distributed between the solid surface and the edge of the prismatic layers on all surfaces except the horizontal tail plane (HTP). For the HTP 48 grid points were used.

The objective of this part of the work is the compilation of first experiences and knowledge with regard to the behaviour of the transition prediction procedure and the outcome that it produces when it is applied to a complex aerodynamic configuration of industrial relevance while using different application modes and, thus, different prediction approaches. Hence, the content of this section has characteristics of a feasibility study and is not meant as a physical validation of the prediction tools used. The documentation of the results forms a basis for Best Practice

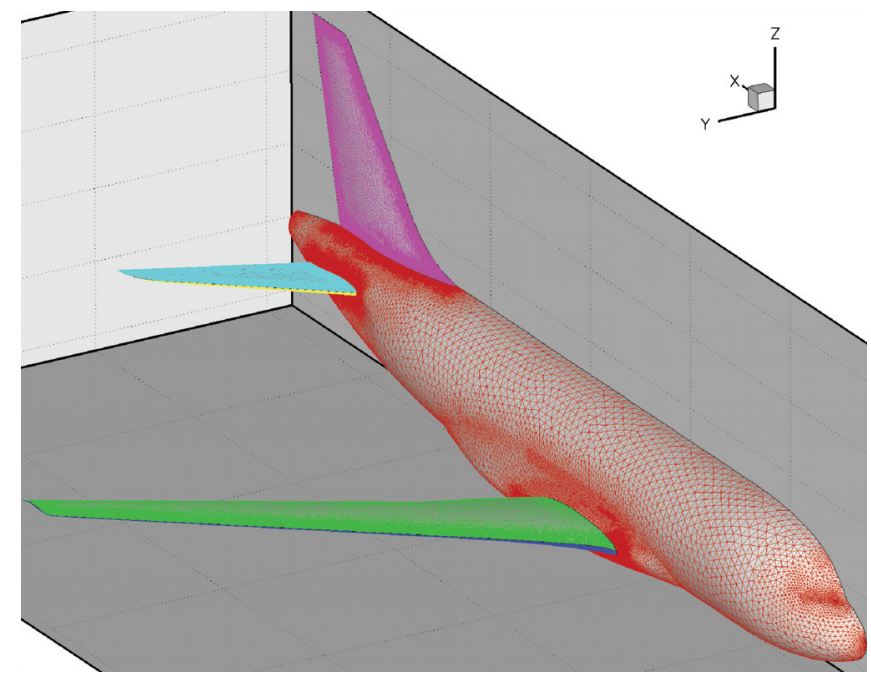

Fig. 13 Surface grid of the three-dimensional generic full aircraft configuration. guidelines necessary for the application of the procedure.

The computations were carried out for the Reynolds number $R e_{\infty}=2.3 \times 10^{6}$ based on the mean aerodynamic chord length of the wing, the Mach number $M_{\infty}=0.2$ and the angle of attack $\alpha=-4.0 \mathrm{deg}$ using the SAE turbulence model. The pitching angle $i_{\mathrm{H}}$ of the HTP with respect to the longitudinal axis is $i_{\mathrm{H}}=4.0 \mathrm{deg}$, so that the local angle of attack of the HTP is $\alpha_{\mathrm{H}}=0.0 \mathrm{deg}$. For the stability analysis using the linear stability code LILO, the critical $N$ factors $N^{T S}{ }_{\text {crit }}=7.0$ and $N^{C F}$ crit $=7.0$ for the detection of TS and CF instabilities were applied. The numerical values for both critical $N$ factors are an arbitrary choice for these test computations. The computations were run parallel on a $2.2 \mathrm{GHz}$ Opteron Linux cluster with $328 \mathrm{CPUs}$ altogether. The computations were performed using 32, 48, or 64 processes.

In the following, the results using BL mode 2 (calculation of inviscid streamlines and laminar boundary-layer parameters from the current flow solution in the RANS grid) and BL mode 1 ( $c_{\mathrm{p}}$-extraction along 'line-in-flight' cuts and calculation of the laminar boundary-layer parameters by the laminar boundary-layer code) are shown and described. Transition was predicted on the upper and lower sides of the HTP using 11 streamlines or 'line-in-flight' cuts, respectively, on each side. The transition prediction iteration was started with a pre-prediction phase of 500 RANS cycles determining laminar separation points and using them as transition points every 20 cycles. Then, every 
500 cycles the transition prediction module was called, and the complete RANS computation was stopped at cycle $k_{c y c}=2500$, that is, the transition module was called four times.

In Fig. 14, the skin friction distribution from the converged RANS computation for the upper and lower sides, the corresponding skin friction lines (lines with arrowheads) and the inviscid streamlines (white lines without arrowheads) from the first and the last call of the transition module are shown. In Fig. 15, the $c_{\mathrm{p}}$-distribution from
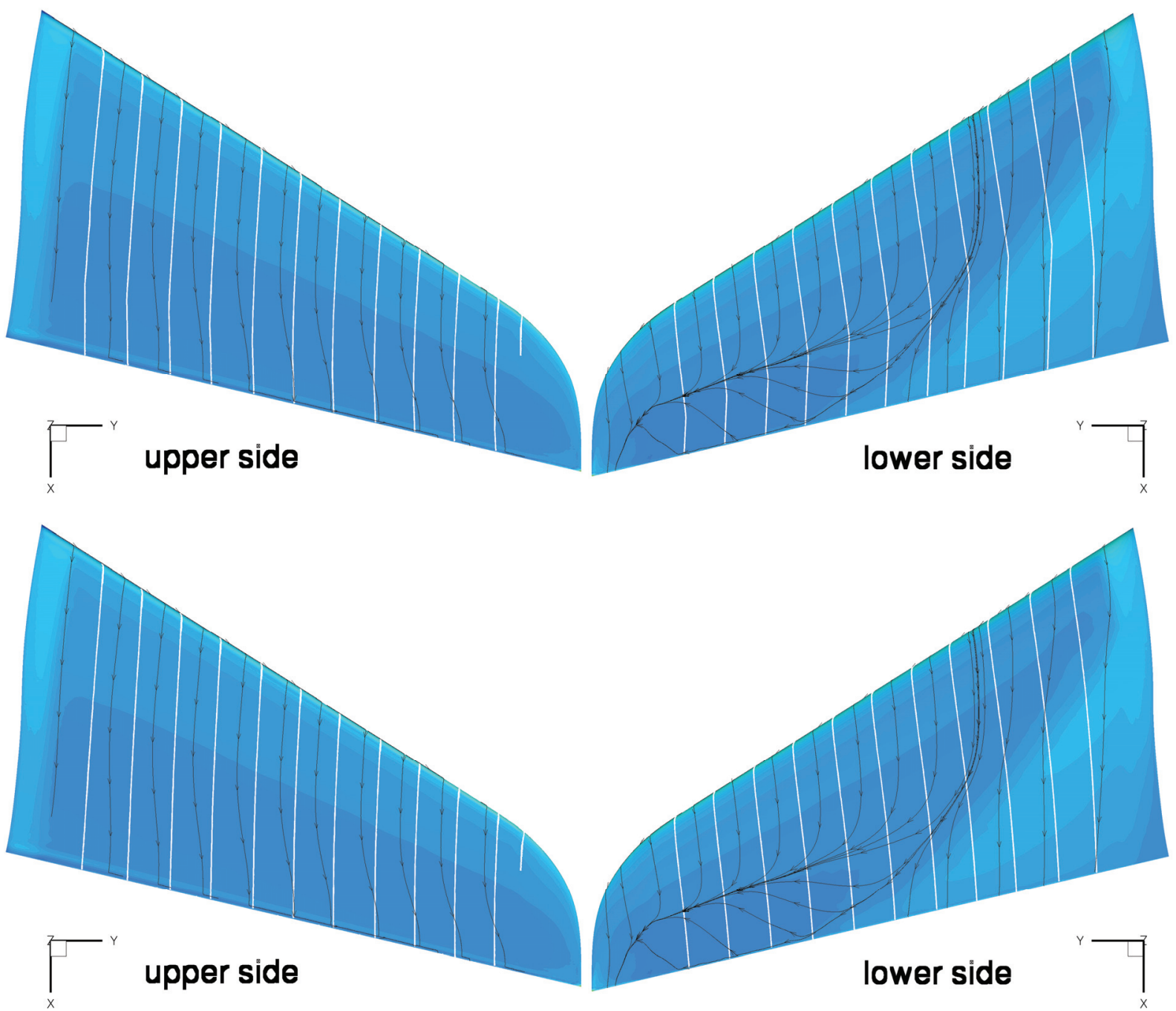

Fig. 14 Skin friction distribution, skin friction lines (lines with arrowheads) and inviscid streamlines (white lines without arrowheads) from first (above) and last (below) call of the transition module, BL mode 2.

the converged RANS computation for the upper and lower sides, the corresponding skin friction lines (lines with arrowheads) and the transition lines (thick lines with symbols) from the first and the last call of the transition module are shown, and in Fig. 16 all transition lines from the four consecutive calls of the transition module. Comparing the running of the inviscid streamlines from the first and the last transition prediction step with the skin friction lines, which are identical in both cases, shows that the inviscid streamlines have not changed significantly during the 1500 RANS cycles, which lie in between. The transition lines move from a more upstream to more downstream position. They start from a situation defined by a line which consists of points detected as laminar separation points during the pre-prediction phase. This intermediate stage of the transition prediction iteration is not shown in these figures. The transition line on the upper side is continuously moving towards the trailing edge and settles there. The transition line on the lower side steps back in upstream direction in the last transition prediction iteration step and probably has not completely settled yet, but the convergence state is good and an advancing influence or a further change due to a 

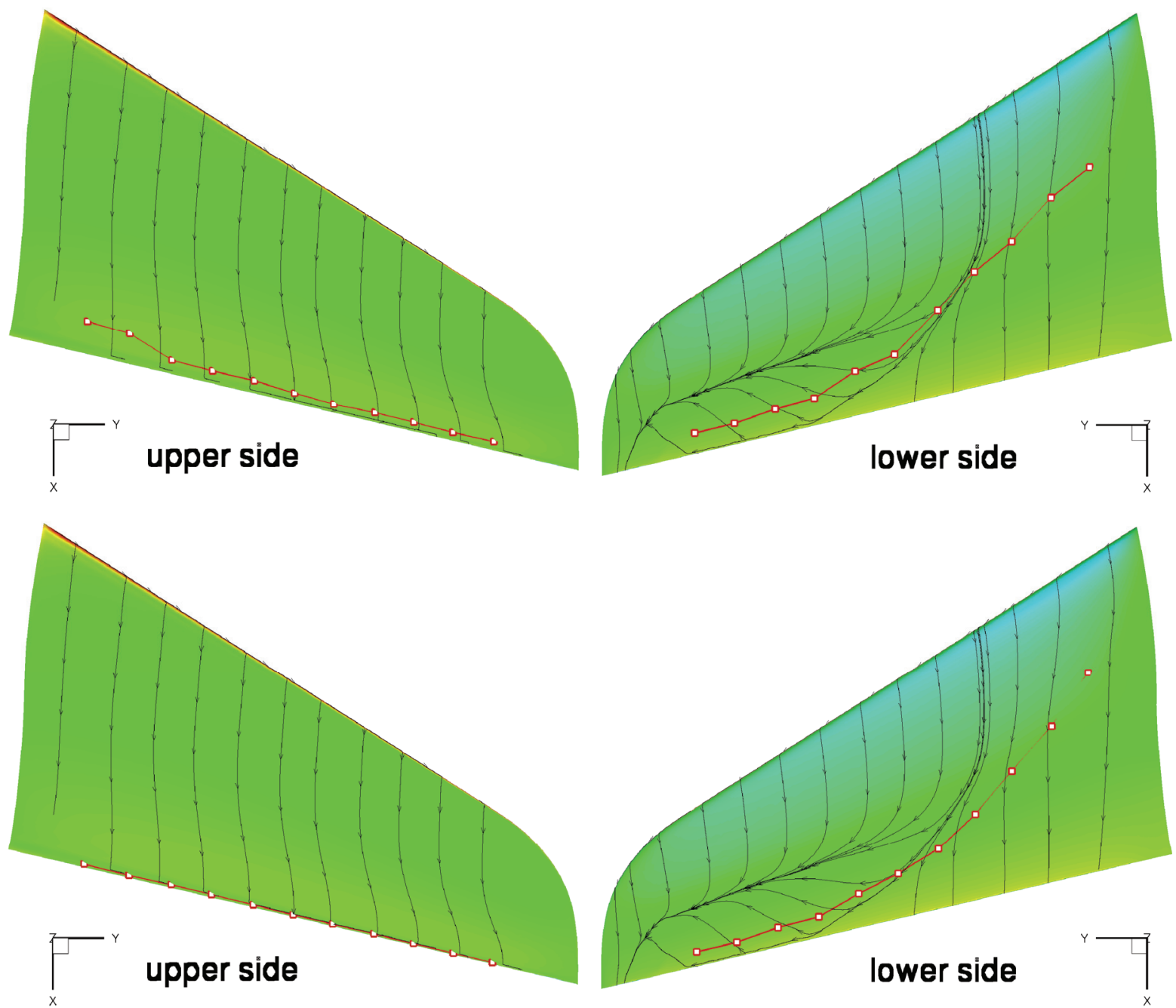

Fig. $15 c_{\mathrm{p}}$-distribution, skin friction lines and transition lines from first (above) and last (below) call of the transition module, BL mode 2.

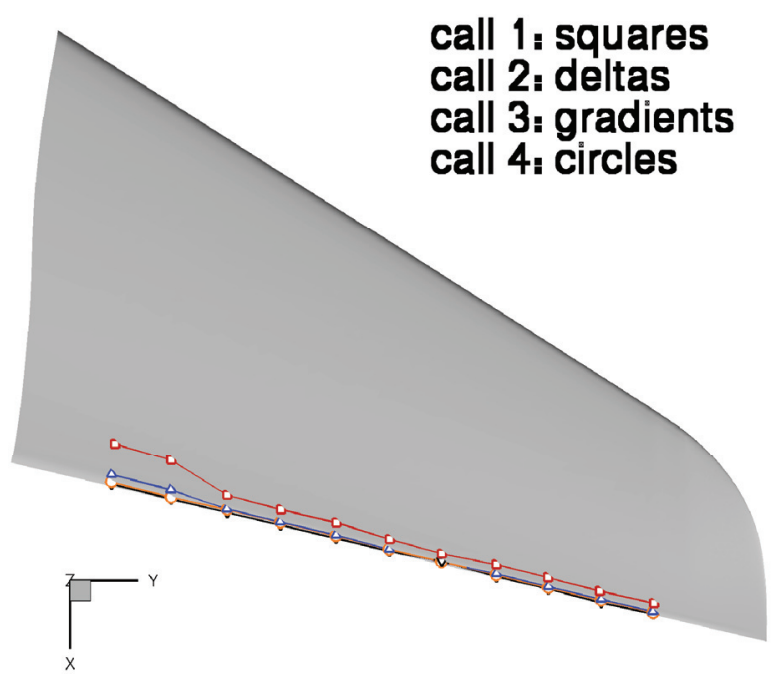

$$
\begin{aligned}
& \text { call 1: squares } \\
& \text { call 2: deltas } \\
& \text { call 3: gradients } \\
& \text { call 4: circles }
\end{aligned}
$$

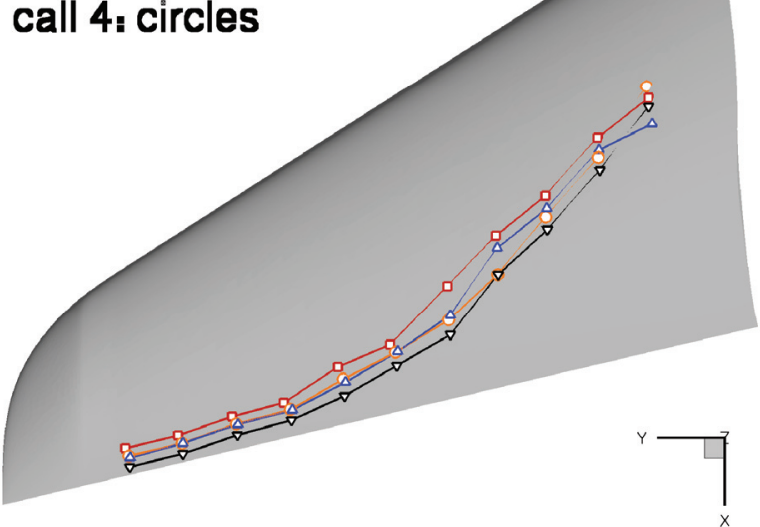

Fig. 16 Transition lines from all calls of the transition module, BL mode 2. 
further settling of the transition line can not be expected. The global RANS convergence history of the coupled computation shown in Fig. 17 shows a well converged state without a clearly visible impact of the changing transition lines at the RANS cycles $k_{c y c}=500,1000,1500$, and 2000 . On the upper side, not a single transition point was found by the stability code due to an instability. During the computation, the complete upper side transition line is moved towards the trailing edge, because along all calculated inviscid streamlines the flow is stable. On the lower side, all transition points were found by the stability code at the end of the transition prediction iteration, because along all the streamlines on the lower side the critical TS $N$ factor was reached. For the two transition points nearest to the wing root of the HTP, also amplified $\mathrm{CF}$ waves exceeding the critical CF $N$ factor were found. These transition locations, however, are located significantly further downstream and, thus,

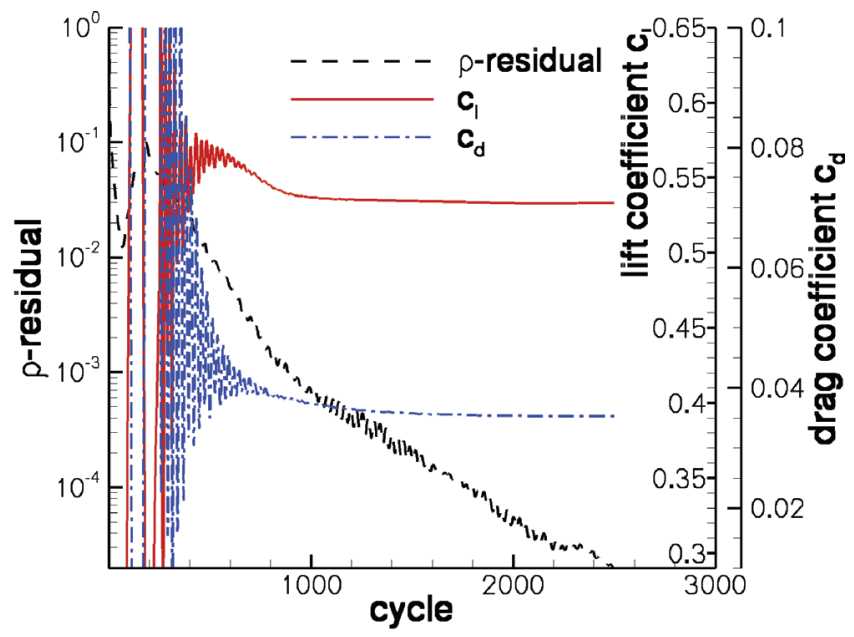

Fig. 17 RANS convergence history for the threedimensional generic full aircraft configuration with predicted transition on the HTP, BL mode 2. were not taken into account by the procedure.

The next figures document the differences which are obtained when BL mode 1 is applied. In Figures 18 to 20 , essentially the same quantities as before are shown. Fig. 18 shows the skin friction distribution from the converged RANS computation for the upper and lower sides of the HTP, the corresponding skin friction lines (lines with arrowheads) and the line-in-flight cuts (white lines without arrowheads). In Fig. 19, the $c_{\mathrm{p}}$-distribution from the converged RANS computation for the upper and lower sides, the corresponding skin friction lines (lines with arrowheads) and the transition lines (thick lines with symbols) from the first and the last call of the transition module are shown, and in Fig. 20 all transition lines from the four consecutive calls of the transition module.
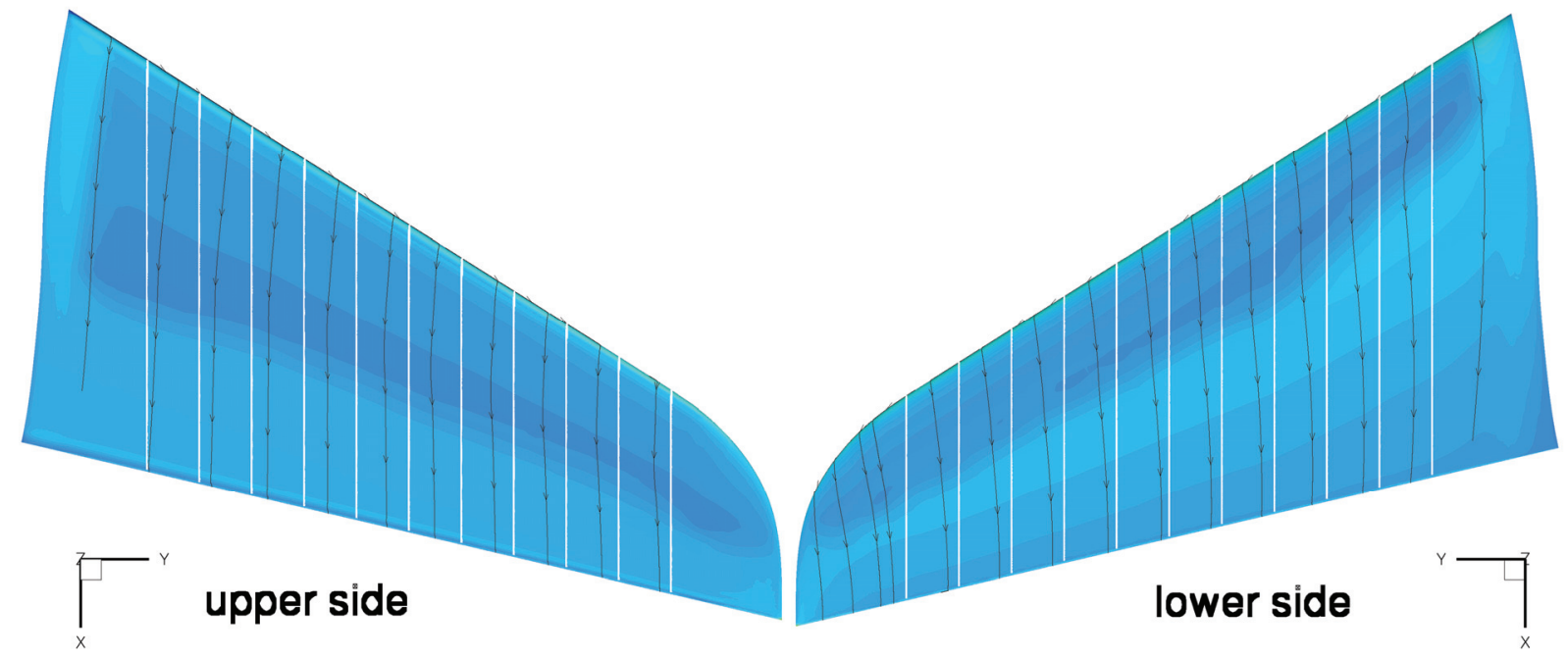

Fig. 18 Skin friction distribution, skin friction lines (lines with arrowheads) and line-in-flight cuts (white lines without arrowheads), BL mode 1.

A comparison of the line-in-flight cuts and the inviscid streamlines from the last transition module call using BL mode 2 shows only minor differences between the two approaches for this test case and, thus, justifies the use of simple sections through the wing for a case of a high aspect ratio wing with no or only moderate flow separation. The running of the skin friction lines on both sides of the HTP, however, is significantly different from the approach using BL mode 2 because of the different locations of the transition lines on both sides. Due to the use of the laminar 

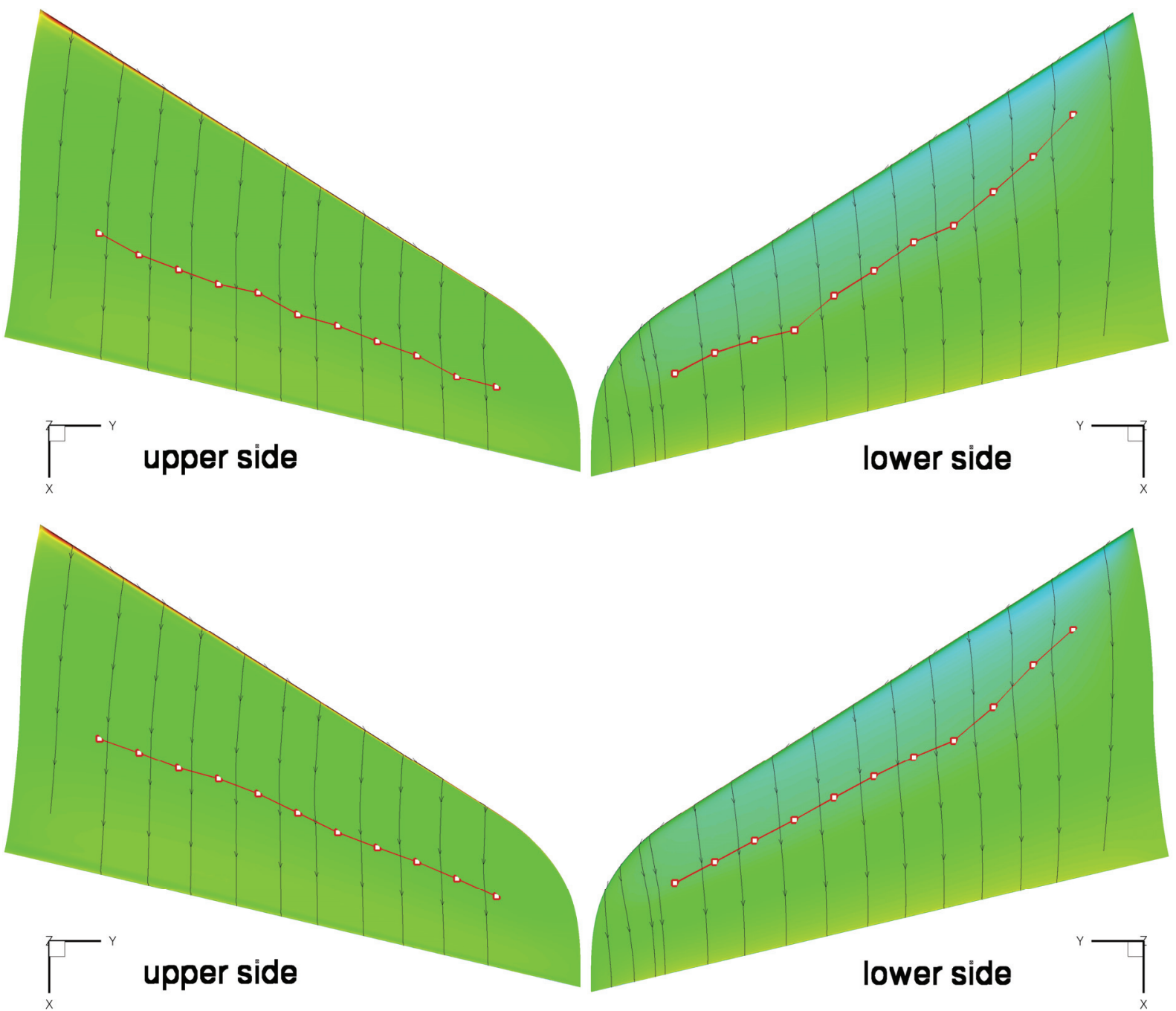

Fig. $19 c_{\mathrm{p}}$-distribution, skin friction lines and transition lines from first (above) and last (below) call of the transition module, BL mode 1.
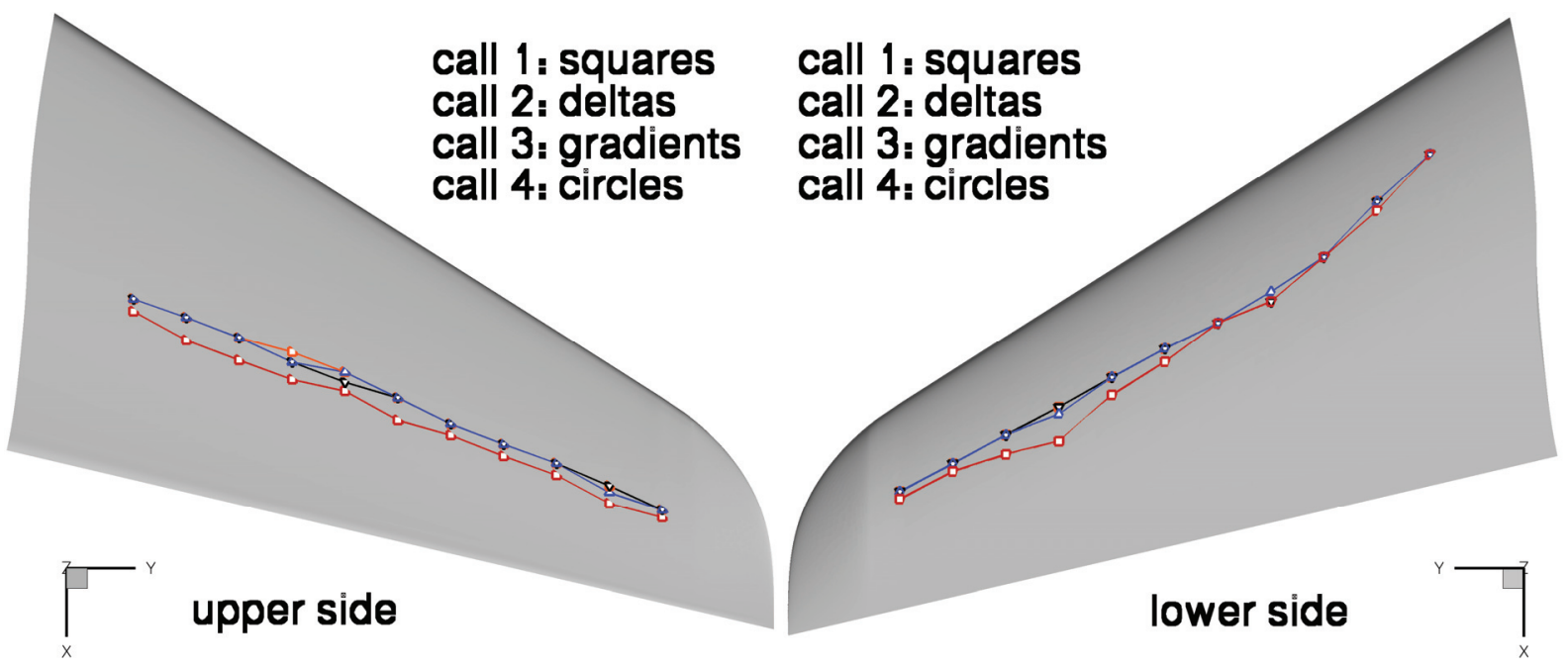

Fig. 20 Transition lines from all calls of the transition module, BL mode 1. 
boundary-layer code the movement of the transition lines proceeds now in the opposite direction compared to BL mode 2. First, as known from other investigations, the laminar separation points determined by the boundary-layer code are located further upstream than those detected in the RANS solution. Except the three transition points which are located most inboard on the lower side of the HTP and which are caused by TS instabilities, all others are approximated by the laminar separation points from the boundary-layer code. Because there are no laminar boundarylayer data available downstream of the laminar separation points, the transition lines can not be shifted into those downstream regions where the disturbances are sufficiently strongly amplified. As a consequence, the critical $N$ factors are not reached. Because of the fact that the lower side transition line is almost completely composed of points which were detected as separation points by the laminar boundary-layer code, the flow separation on the HTP lower side that occurs in the simulation using BL mode 2 can not develop here. Directly downstream of the transition line the turbulence model becomes active, the boundary layer is enriched with energy by the increasing level of turbulence and stays attached. After the last call of the transition module, both transition lines are in a well converged state. The corresponding RANS convergence history of the computation using $\mathrm{BL}$ mode 1 is depicted in Fig. 21, where it is compared to the RANS convergence history of the computation using BL mode 2 (thin lines). As expected, the convergence proceeds faster and with much lower oscillations than those from the computation using BL mode 2. A close inspection of the two sets of lines reveals that the lift coefficients of both simulations reach almost the same value at the end of the computations. The values of the converged drag coefficients, however, differ slightly. The drag value of the computation using BL mode 1 is visibly higher than the one from the computation using BL mode 2.

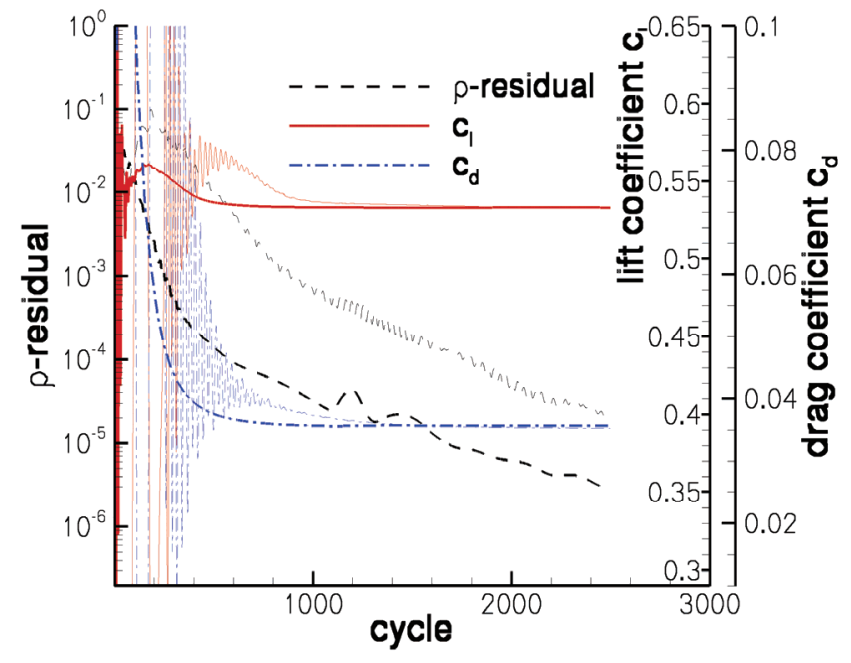

Fig. 17 RANS convergence history for the threedimensional generic full aircraft configuration with predicted transition on the HTP, BL mode 1.

With regard to free flight simulations, the employment of BL mode 2 may prove to be more adequate to predict transition at the HTP for this and similar flow cases. For free flight conditions and, thus, for design purposes in the aircraft industry the results from the ATTAS/VFW-614 and Fokker F100 free flight experiments are available [36], [47]-[48]. There, for Tollmien-Schlichting transition $N_{\text {crit }}^{T S} \approx 12$ and for cross flow transition $N^{C F}$ crit $\approx 9$ can be read. Both values are significantly higher than the values used in the present test computations, $N_{\text {crit }}^{T S}=N_{\text {crit }}^{C F}=7.0$, so that one can assume that the approximation of transition points by using laminar separation points would lead to an erroneous result. Whether this assumption is true, must be verified in the future using a finer grid resolution in the prismatic layers of the HTP, so that also the cross flow velocity profiles are reproduced accurately enough. Not until this prerequisite is fulfilled, one can fully rely on the internal calculation of the laminar boundary-layer parameters and the outcome of the cross flow stability analysis performed with the linear stability code based on these data.

Much more test computations using the transition prediction module of the TAU code will be carried out in the nearest future, in order to fully assess its capabilities, its characteristics and its behaviour. Some of the next steps will be the investigation of the transition behaviour at the HTP of the three-dimensional generic full aircraft configuration for different angles of attack and for different values of the critical $N$ factors and the application of the transition prediction procedure to all wings of the aircraft. Finally, also the fuselage will be included.

\section{Conclusion}

A hybrid Reynolds-averaged Navier-Stokes solver with an automatic transition prediction functionality was successfully applied to a two-dimensional two-element airfoil configuration and a three-dimensional generic full aircraft configuration which are both of industrial relevance. The RANS solver which is coupled to a transition prediction module represents a simulation system that predicts the unknown transition locations during the ongoing RANS computation iteratively in an automatic manner without intervention of the user of the code. The transition prediction module contains a laminar boundary-layer code for swept, tapered wings and compressible, conical external flow and a fully automated local, linear stability code for the prediction of transition due to TollmienSchlichting and cross flow instabilities using the $\mathrm{e}^{N}$-method based on the two $N$ factor approach. 
The two configurations were investigated applying the automatic transition prediction procedure using different application modes. The one mode is based on the internal calculation of the laminar boundary-layer parameters from the intermediate flow solutions in the RANS computational grid, the other mode makes use of the laminar boundarylayer code for the calculation of all laminar boundary-layer viscous data based on the surface pressure distribution from the RANS solution. The handling of the different modes was described and the results of their application were compared. For the two-element airfoil configuration, numerical instability problems which may arise when transition inside laminar separation bubbles is to be predicted were discussed in detail.

It was shown that the coupled system can be applied to three-dimensional aircraft configurations and that it can be used by the design engineer for flow problems in which laminar-turbulent transition plays a role without $a$ priori knowledge of the transition characteristics. The documentation of the results forms a basis for Best Practice guidelines necessary for the application of the procedure.

\section{Acknowledgments}

A. Krumbein and N. Krimmelbein thank Airbus for providing the computational grid of the two-dimensional twoelement airfoil configuration and acknowledge Airbus for letting them use the geometry of the three-dimensional generic full aircraft configuration. The authors thank Mr. M. Kruse, Institute of Fluid Mechanics, Technical University of Braunschweig for providing the computational grid of the three-dimensional generic full aircraft configuration.

\section{References}

[1] Rudnik, R., Ronzheimer, A., and Schenk, M., „Berechnung von zwei- und dreidimensionalen Hochauftriebskonfigurationen durch Lösung der Navier-Stokes Gleichungen“, Jahrbuch 1996 - Bd. II der Deutschen Gesellschaft für Luft- und Raumfahrt - Lilienthal-Oberth e.V. (DGLR) - JT 96-104, Deutscher Luft- und Raumfahrtkongreß, DGLR-Jahrestagung Sep. 1996, Dresden, Deutsche Gesellschaft für Luft- und Raumfahrt - LilienthalOberth e.V. (DGLR), Godesberger Allee 70, D-53175 Bonn, Germany, 1996, pp. 717-726.

[2] Smith, A.M.O., Gamberoni, N., "Transition, Pressure Gradient and Stability Theory“, Douglas Aircraft Company, Long Beach, Calif. Rep. ES 26388, 1956.

[3] van Ingen, J.L., "A suggested Semi-Empirical Method for the Calculation of the Boundary Layer Transition Region", University of Delft, Dept. of Aerospace Engineering, Delft, The Netherlands, Rep. VTH-74, 1956.

[4] Arnal, D., Casalis, G., "Laminar-turbulent transition prediction in three-dimesnional flows", Progress in Aerospace Sciences, Vol. 36, 2000, pp. 173-191.

[5] Radespiel, R., Graage, K., Brodersen, O., "Transition Predictions Using Reynolds-Averaged Navier-Stokes and Linear Stability Analysis Methods“, AIAA Paper 91-1641, 1991.

[6] Stock, H. W., Haase, W., "A Feasibility Study of $\mathrm{e}^{N}$ Transition Prediction in Navier-Stokes Methods for Airfoils", AIAA Journal, Vol. 37, no. 10, 1999, pp. 1187-1196.

[7] Horton, H. P., Stock, H. W., "Computation of Compressible, Laminar Boundary Layers on Swept, Tapered Wings", Journal of Aircraft, Vol. 32, 1995, pp. 1402-1405.

[8] Stock, H. W., Degenhardt, E., "A simplified $\mathrm{e}^{N}$ method for transition prediction in two-dimensional, incompressible boundary layers“, Zeitung für Flugwissenschaft und Weltraumforschung, Vol. 13, 1989, pp. 16-30.

[9] Casalis, G., Arnal, D., "ELFIN II Subtask 2.3: Database method - Development and validation of the simplified method for pure crossflow instability at low speed”, ELFIN II - European Laminar Flow Investigation, Technical Report $\mathrm{n}^{\circ} 145$, ONERA-CERT, Département d'Études et de Recherches en Aérothermodynamique (DERAT), R.T. DERAT n ${ }^{\circ}$ 119/5618.16, December 1996.

[10] Crouch, J. D., Crouch, I. W. M., Ng, L. L., "Transition Prediction for Three-Dimensional Boundary Layers in Computational Fluid Dynamics Applications“, AIAA Journal, Vol. 40, no. 8, 2002, pp. 1536-1541.

[11] Warren, E. S., Hassan, H. A., "Transition Closure Model for Predicting Transition Onset", Journal of Aircraft, Vol. 35, 1998, pp. 769-775.

[12] Czerwiec, R.M., Edwards, J. R., Rumsey, C. L., Bertelrud, A., Hassan, H. A., "Study of High-Lift Configurations Using k$\omega$ Transition/Turbulence Model“", AIAA Paper 99-3186, 1999.

[13] Edwards, J. R., Roy, C. J., Blottner, F. G., Hassan, H. A., "Development of a One-Equation Transition/Turbulence Model“", AIAA Journal, Vol. 39, no. 9, 2001, pp. 1691-1698.

[14] Langtry, R.B., Menter, F.R., "Transition Modeling for General CFD Applications in Aeronautics", AIAA Paper 2004-522, 2005.

[15] Radespiel, R., Windte, J., Scholz, U., "Numerical and Experimental Analysis of Moving Airfoils with Laminar Separation Bubbles", AIAA Paper 2006-0501, 2006.

[16] Windte, J., Scholz, U., Radespiel, R., "Validation of the RANS-Simulation of Laminar Separation Bubbles on Airfoils", Aerospace Science and Technology, Vol. 10, No. 6, 2006, pp. 484-494.

[17] Wokoeck, R., Krimmelbein, N., Ortmanns, J., Ciobaca, V., Radespiel, R., Krumbein, A., "RANS Simulation and Experiments on the Stall Behaviour of an Airfoil with Laminar Separation Bubbles", AIAA Paper 2006-244, 2006. 
[18] Krumbein, A., Krimmelbein, N., "Navier-Stokes High-Lift Airfoil Computations with Automatic Transition Prediction using the DLR TAU Code", 15. DGLR-Fach-Symposium der STAB, Technical University of Darmstadt, Germany, 29 November - 1 December 2006, accepted for publication in New Results in Numerical and Experimental Fluid Mechanics, Notes on Numerical Fluid Mechanics and Multidisciplinary Design, Springer Verlag, Berlin.

[19] Krumbein, A., "Automatic Transition Prediction and Application to High-Lift Multi-Element Configurations", Journal of Aircraft, Vol. 42, No. 5, 2005, pp. 1150-1164; also: AIAA Paper 2004-2543, June/July 2004.

[20] Krumbein, A., "Automatic Transition Prediction and Application to Three-Dimensional Wing Configurations", Journal of Aircraft, Vol. 44, No. 1, 2007, pp. 119-133; also: AIAA Paper 2006-914, January 2006.

[21] Krumbein, A., "Automatic Transition Prediction and Application to 3-D High-Lift Configurations", in print at Journal of Aircraft, DOI: 10.2514/1.25528; also: AIAA-2006-3164, June 2006.

[22] Stock, H.W., "e $\mathrm{N}^{\mathrm{N}}$ Transition Prediction in Three-Dimensional Boundary Layers on Inclined Prolate Spheroids", AIAA Journal, Vol. 44, No. 1, 2006, pp. 108-118.

[23] Arthur, M.T., Atkin, C.J., "Transition modelling for viscous flow prediction”, AIAA Paper 2006-3052, 2006.

[24] Krimmelbein, N., Radespiel, R., Nebel, C., "Numerical Aspects of Transition Prediction for Three-Dimensional Configurations", AIAA-2005-4764.

[25] TAU-Code User Guide, Release 2007.1.0, Institute of Aerodynamics and Flow Technology, German Aerospace Center, April 2007.

[26] Schwamborn, D., Gerhold, T., Hannemann, V., "On the Validation of the DLR-TAU Code", New Results in Numerical and Experimental Fluid Mechanics II, Notes on Numerical Fluid Mechanics, Vol. 72, Braunschweig, Wiesbaden, Vieweg Verlag, 1999, pp. 426-433.

[27] Kroll, N., Rossow, C.-C., Schwamborn, D., Becker, K., and Heller, G., "MEGAFLOW - A Numerical Flow Simulation Tool For Transport Aircraft Design", ICAS Congress 2002 [CD-Rom], ICAS, Toronto, Canada, 2002, pp. 1.105.11.105.20.

[28] Schwamborn, D., Gerhold, T., Heinrich, R., "The DLR TAU-Code: Recent Applications in Research and Industry", European Conference on Computational Fluid Dynamics, ECCOMAS CFD 2006, Egmond aan Zee, The Netherlands, $5-8$ September 2006, ECCOMAS CFD 2006 - CD-Rom Proceedings, editors: P. Wesseling, E. Oñate, J. Périaux, 2006, ISBN: 90-9020970-0, (C) TU Delft, The Netherlands.

[29] Nebel, C., Radespiel, R., and Wolf, T., "Transition Prediction for 3D Flows Using a Reynolds-Averaged Navier-Stokes Code and N-Factor Methods", AIAA-2003-3593.

[30] Krimmelbein, N., Radespiel, R., "Transition prediction for three-dimensional flows using parallel computation", submitted to Computers \& Fluids, Elsevier, March 2007.

[31] "COCO - A Program to compute Velocity and Temperature Profiles for Local and Nonlocal Stability Analysis of Compressible, Conical Boundary Layers with Suction”, ZARM Technik Report, November 1998.

[32] Schrauf, G., "LILO 2.1 User's Guide and Tutorial", Bremen, Germany, GSSC Technical Report 6, originally issued Sep. 2004, modified for Version 2.1 July 2006.

[33] Rozendaal, R. A., "Natural Laminar Flow Flight Experiments on a Swept Wing Business Jet-Boundary-Layer Stability Analysis", NASA CP 3975, March 1986.

[34] Rozendaal, R. A., "Variable-Sweep Transition Flight Experiment (VSTFE)-Parametric Pressure Distribution BoundaryLayer Stability Study and Wing Glove Design Task”, NASA CR 3992, December 1986.

[35] Rozendaal, R. A., "Variable-Sweep Transition Flight Experiment (VSTFE)-Stability Code Development and Clean-Up Glove Analysis", NASA CP 2847, April 1987.

[36] Schrauf, G., "Large-Scale Laminar-Flow Tests Evaluated with Linear Stability Theory", Journal of Aircraft, Vol. 41, No. 2, March/April 2004, pp. 224 - 230.

[37] Stock, H. W., "Infinite Swept Wing RANS Computations with e ${ }^{N}$ Transition Prediction - Feasibility Study", Rept. IB 1242003/12, Deutsches Zentrum für Luft- und Raumfahrt (DLR), Braunschweig, Germany, Aug. 2002.

[38] Krumbein, A., "Navier-Stokes Airfoil Computations with Automatic Transition Prediction using the DLR TAU Code - A Sensitivity Study“, New Results in Numerical and Experimental Fluid Mechanics 5, Notes on Numerical Fluid Mechanics and Multidisciplinary Design, Vol. 92, Springer Verlag, Berlin, 2006, pp. 192-199.

[39] Krumbein, A., "Automatic Transition Prediction for High-Lift Systems Using a Hybrid Flow Solver", Journal of Aircraft, Vol. 42, No. 5, 2005, pp. 1362-1366.

[40] Schmidt, G. S., Mueller, Th. J., "Analysis of Low Reynolds Number Separation Bubbles Using Semiempirical Methods“, AIAA Journal, Vol. 27, No. 8, 1989, pp. 993-1001.

[41] Davis, R. L., Carter, J. E., Reshotko, E., "Analysis of Transitional Separation Bubbles on Infinite Swept Wings", AIAA Journal, Vol. 25, No. 3, March 1987, pp. 421-428.

[42] Stock, H. W., Haase, W., "Some Aspects of Linear Stability Calculations in Industrial Applications", Transitional Boundary Layers in Aeronautics, Proceedings of the colloquium 'Transitional Boundary Layers in Aeronautics', Amsterdam, 6-8 December 1995, editors: Henkes, R. A. W. M.; van Ingen, J.L., Royal Netherlands Academy of Arts and Sciences, P.O. Box 1912, 1000 GC Amsterdam, The Netherlands, North-Holland, Amsterdam/Oxford/New-York/Tokyo, ISBN: 0-444-85812-1, 1996, pp. 225-238.

[43] van den Berg, B., "Boundary Layer Measurements on a Two-Dimensional Wing with Flap", National Aerospace Laboratory - NLR, Amsterdam, The Netherlands, NLR TR 79009 U, January 1979.

[44] Spalart, P. R., and Allmaras, S. R., “A one-equation turbulence model for aerodynamic flows“, La Recherche Aérospatiale, 1994, n 1, pp. 5-21. 
[45] Edwards, J.R., and Chandra, S., "Comparison of Eddy Viscosity-Transport Turbulence Models for Three-Dimensional, Shock-Separated Flowfields“, AIAA Journal, Vol. 34, No. 4, 1996, pp. 756-763.

[46] Krumbein, A., " $\mathrm{e}^{\mathrm{N}}$ Transition Prediction for 3D Wing Configurations using Database Methods and a local, linear Stability Code", 7th ONERA-DLR Aerospace Symposium - ODAS 2006, 4 - 7 October 2006, Toulouse, France, ODAS 2006 - CDROM Proceedings, editor: ONERA, Centre de Toulouse, October 2006 (submitted to Aerospace Science and Technology).

[47] Schrauf, G., Perraud, J., Lam, F., "Comparison of Boundary-Layer Transition Predictions Using Flight Test Data“, Journal of Aircraft, Vol. 35, No. 6, 1998, pp. 891-897.

[48] Schrauf, G., Atkin, Ch., "Progress in Linear Stability Methods for Design Applications“, ECCOMAS 2000, Barcelona (e), 11.-14. September 2000, ECCOMAS 2000 - CD-Rom Proceedings, editor: International Center for Numerical Methods in Engineering (CIMNE), 2000, ISBN: 84-89925-70-4, Depósito Legal: B-37139-2000. 